\title{
NON-SINGULAR MULTILINEAR FORMS AND CERTAIN $p$-WAY MATRIX FACTORIZATIONS*
}

\author{
BY \\ RUFUS OLDENBURGER
}

1. Introduction. Let there be given a $p$-way matrix $A=\left(a_{i_{1}} \ldots i_{p}\right)$, $i_{1}, \cdots, i_{p}=1, \cdots, n$. The operation which takes $A$ into $A^{\prime}=\left(a_{i_{1}} \cdots i_{p} b_{i_{1} j_{1}}\right)$, where $B=\left(b_{i_{1} j_{1}}\right)$ is a non-singular 2-way matrix and the repeated index indicates summation, is called a non-singular linear transformation on the index $i_{1}$ of $A$ with the matrix $B$. It is also said to be a non-singular linear transformation on $A$. If a matrix $A^{\prime}$ is obtained from $A$ by making nonsingular linear transformations on the indices of $A$ with matrices having elements in a field $\phi$, then $A^{\prime}$ is said to be equivalent in the field $\phi$ to $A$. If $A$ and $A^{\prime}$ are 2-way matrices this is equivalence in the ordinary sense.

The matrix $A$ is said to be non-singular if $A$ is equivalent in some field $\phi$, where $\phi$ contains the elements of $A$, to $\delta=\left(\delta_{j_{1}} \cdots j_{p}\right)$, where $\delta_{j_{1} \cdots j_{p}}=1$ for $j_{1}=\cdots=j_{p}=1, \cdots, n$, and $\delta_{j_{1}} \cdots j_{p}=0$ if $\left(j_{1}, \cdots, j_{p}\right) \neq(1, \cdots, 1), \cdots$, $(n, \cdots, n)$. Similarly a $p$-way multilinear form

$$
G=a_{i_{1} \cdots i_{p}} y_{i_{1}}^{(1)} \cdots y_{i_{p}}^{(p)} \quad\left(i_{1}, \cdots, i_{p}=1, \cdots, n\right)
$$

is said to be non-singular if $G$ is equivalent under non-singular linear transformations

$$
y_{i_{1}}^{(1)}=b_{i_{1} j_{1}}^{(1)} x_{j_{1}}^{(1)}, \cdots, y_{i_{p}}^{(p)}=b_{i_{p} i_{p}}^{(p)} x_{j_{p}}^{(p)} \quad\left(j_{1}, \cdots, j_{p}=1, \cdots, n\right)
$$

to

$$
F=\sum_{j_{1}=\cdots=j_{p}=1}^{n} x_{i_{1}}^{(1)} \cdots x_{j_{p}}^{(p)} .
$$

In chapter I of this paper sets of necessary and sufficient conditions, which may be applied in a finite number of steps to a given matrix, are derived for a $p$-way matrix $A$, and therefore for its associated form $G$, to be non-singular. It is necessary in the treatment to distinguish between the cases $\dagger$ where $p=3$ and $p \geqq 4$. Among necessary and sufficient conditions for non-singularity it is proved that a matrix $A$ as given above is non-singular if and only if $A$ can be

* Presented to the Society, March 30 and April 6 and 7, 1934. Abstracts appeared in the Bulletin of the American Mathematical Society, vol. 40 (1934), pp. 219, 226, 227, under the numbers 140, 164, 165 respectively. Received by the editors March 22, 1935.

$\dagger$ The treatment of the case $p=2$ is assumed known. 
"factored" into the form $\left(c_{\alpha i_{1}}^{(1)} \cdots c_{\alpha i_{p}}^{(p)}\right)$, where $\left(c_{\alpha i_{1}}^{(1)}\right), \cdots,\left(c_{\alpha i_{p}}^{(p)}\right)$ are non-singular 2-way matrices.

The factorization property of a non-singular matrix $A$ suggests the more general problem of determining the conditions under which a matrix can be written in the form $\left(c_{\alpha i_{1}}^{(1)} \cdots c_{\alpha i_{p}}^{(p)}\right), \alpha, i_{1}, \cdots, i_{p}=1, \cdots, n$, where $\left(c_{\alpha i_{1}}^{(1)}\right)$ is singular, and $\left(c_{\alpha i_{2}}^{(2)}\right), \cdots,\left(c_{\alpha i_{p}}^{(p)}\right)$ are non-singular. ${ }^{*}$

In the factorizations mentioned above the index $\alpha$ is summed. Necessary and sufficient conditions are also obtained (chapter III) for a matrix $B=\left(b_{\alpha i_{1}} \cdots i_{p}\right)$ to be of the form $\left(a_{\alpha \beta i_{1}}^{(1)} \cdots a_{\alpha \beta i_{p}}^{(p)}\right)$, $\alpha$ not summed, where the 3-way matrices $\left(a_{\alpha \beta i_{1}}^{(1)}\right), \cdots,\left(a_{\alpha \beta i_{p}}^{(p)}\right)$ are non-singular if arrayed as 2-way matrices with $\alpha \beta$ as the row index. The method of treatment applies to the case where $B=\left(b_{i_{1}} \cdots i_{p}\right)$ factors into the form $\left(c_{\alpha_{i_{1}}}^{(1)} \cdots c_{\alpha i_{p}}^{(p)}\right)$, where $\alpha$ is not summed, and $\left(c_{\alpha_{1}}^{(1)}\right), \cdots,\left(c_{\alpha i_{p}}^{(p)}\right)$ are non-singular.

The terminology and notations used in the ordinary theory of 2-way matrices are assumed known to the reader.

The paper is divided as follows: $\$ 1$, Introduction. Chapter I, Non-singular multilinear forms: §2, Definitions; §3, Similar transformations; §4, Preliminary theorems; $\S 5$, Necessary and sufficient conditions for a matrix to be non-singular; $\S 6$, Note on invariant factors. Chapter II, Factorization of $p$-way matrices into a product of 2-way matrices one of which is singular: $\S 7$, Introduction; §8, Canonical diagonal 2-way matrices; §9, Necessary and sufficient conditions for the equivalence of a set of 2-way matrices to a set of diagonal matrices; $\$ 10$, Necessary and sufficient conditions for the equivalence of a set of $p$-way matrices, $p \geqq 3$, to a set of diagonal matrices. Chapter III, Factorization of $p$-way matrices into 3-way matrices: $\$ 11$, Introduction; $\S 12$, Factorization into multiple composites.

\section{Chapter I. Non-Singular MUltilinear Forms}

2. Definitions. The number of elements in the range of an index $i$ is said to be the order of $i$. Thus if $i$ varies over $1,2, \cdots, n$, then $i$ is of order $n$. A matrix is said to be of order $n$ if each index is of order $n$. An ordered set

* In the paper entitled $A$ new method in the theory of quantics, Journal of Mathematics and Physics, vol. 8 (1929), pp. 83-84, Hitchcock shows how a matrix of order $n$ associated with a polyadic can always be factored into a sum of products of 2 -way matrices, i.e.,

$$
\left(a_{i_{1}} \cdots i_{p}\right)=\left(\sum_{j=1}^{h} a_{1 j i_{1}} \cdots a_{p j i_{p}}\right) \text {. }
$$

In his paper entitled The expression of a tensor or a polyadic as a sum of products, Journal of Mathematics and Physics, vol. 6 (1927), pp. 164-189, he considers the problem of finding the values of $n, p, h$ for which a matrix can be factored as above into a sum of products of 2-way matrices. He solves a few special cases, but does not solve the general problem. 
of indices of a matrix is called a partition $\dagger$ of indices. Two partitions $T_{1}, T_{2}$ are said to be equal if they have the same number of indices and corresponding indices are of the same order (the first indices "correspond," the second indices "correspond," etc.). We then write $T_{1}=T_{2}$. The product of the orders of the indices in a partition is called the order of the partition. An asterisk on $T$, where $T$ denotes a partition, indicates that the indices of $T$ have been assigned fixed values. For example, if $T=i j k$, and we assign to $i, j, k$ the values $2,4,3$ respectively, we have $T^{*}=243$. If $T_{1}=T_{2}$ and corresponding indices of $T_{1}$ and $T_{2}$ have been assigned the same fixed values, we write $T_{1}^{*}=T_{2}^{*}$.

Let $T_{1}, T_{2}, \cdots, T_{r}$ be mutually exclusive, exhaustive partitions of the indices of a matrix $A$. The display $\left(a_{T_{1}} \ldots T_{r}\right)$ of $A$ is the matrix obtained by assigning $T_{1}, \cdots, T_{r}$ as indices to the different directions of an $r$-space. The indices in each partition are assumed for convention to vary from right to left; e.g., if $i_{1}=1,2 ; i_{2}=1,2,3 ; T=i_{1} i_{2}$; then $T$ varies over the range $\left(i_{1} i_{2}\right)=(11)$, (12), (13), (21), (22), (23).

The $\left(T_{1}, \cdots, T_{r}\right)$ diagonal elements of a matrix $A=\left(a_{T_{1}} \ldots T_{r}\right)$ in which $T_{1}=T_{2}=\cdots=T_{r}$ are the elements obtained by letting $T_{1}^{*}=T_{2}^{*}=\cdots=T_{r}^{*}$. A matrix $A=\left(a_{i_{1}} \cdots i_{p}\right)$ is said to be a diagonal matrix if its only non-vanishing elements are $\left(i_{1}, \cdots, i_{p}\right)$ diagonal elements.

A $T$-layer of $A$ is a minor of $A$ obtained by fixing the partition $T$ in the sense that the indices of $T$ are assigned fixed values, and letting the indices of $A$ not contained in $T$ vary over their complete ranges. The $T$-rank of $A$ is the number of linearly independent $T$-layers of $A$. A matrix $A$ is said to be non-singular on $T$ if the $T$-layers of $A$ are linearly independent. If a matrix $A=\left(a_{i j k}\right)$ is non-singular on $i j$, and $k$, then $A$ possesses an inverse $\left(A_{k i^{\prime} j^{\prime}}\right)=\left(A_{k^{\prime} i j}\right)$ on $i j, k$, where

$$
\left(a_{i j k} A_{k i^{\prime} j^{\prime}}\right)=\left(\delta_{i j i^{\prime} j^{\prime}}\right) ; \quad\left(a_{i j k} A_{k^{\prime} i j}\right)=\left(\delta_{k k^{\prime}}\right) .
$$

$\left(\delta_{k k^{\prime}}\right)$ is a Kronecker delta. $\left(\delta_{i j i^{\prime} j^{\prime}}\right)$ displayed in the form $\left(\delta_{T T^{\prime}}\right), T=i j$, $T^{\prime}=i^{\prime} j^{\prime}$, is also a Kronecker delta.

A $\delta$-matrix on $\left(T_{1}, \cdots, T_{r}\right)$ is the matrix $\left(\delta_{T_{1}} \ldots T_{r}\right)$, where $T_{1}=T_{2}$ $=\cdots=T_{r}$ and $\delta_{T_{1}} \cdots T_{r}^{*}=1$ when $T_{1}^{*}=T_{2}^{*}=\cdots=T_{r}^{*}$, while $\delta_{T_{1}} * T_{2}{ }^{*} \cdots T_{r^{*}}$ $=0$ otherwise.

The composite on $T$ of the matrices $A=\left(a_{\rho} T\right), B=\left(b_{T_{\sigma}}\right)$, where $\rho, \sigma, T$ are partitions, is defined to be the matrix $A|T| B=\left(a_{\rho T} b_{T \sigma}\right)$. The repetition of the partition $T$ in the last matrix indicates that the indices in $T$ are summed. The matrix $A \times B=\left(a_{i_{1}} \ldots i_{m} b_{j_{1}} \ldots j_{n}\right)$ is called the open product of the matrices $A=\left(a_{i_{1}} \cdots i_{m}\right), B=\left(b_{i_{1}} \cdots j_{n}\right)$. A matrix $A=\left(a_{i j k_{1}}^{(1)} \cdots a_{i j k_{p}}^{(p)}\right), i$ not summed $(j$

$\dagger$ Some of the definitions given in this section are given in Composition and rank of $n$-way matrices and multilinear forms, Annals of Mathematics, vol. 35 (1934), pp. $622-657$. 
summed), is said to be the multiple-composite on the indices $i, j$ of the matrices $\left(a_{i j k_{1}}^{(1)}\right), \cdots,\left(a_{i j k_{p}}^{(p)}\right)$.

A matrix $B=\left(b_{i_{1} \cdots i_{p}}\right)$ is said to be composed of a matrix $A=\left(a_{i_{1}} \cdots i_{p}\right)$, $i_{s}=1, \cdots, n_{s}, s=1, \cdots, p$, bordered by zeros, if $b_{j_{1} \cdots i_{p}}=a_{i_{1} \cdots i_{p}}$ for $j_{s}=i_{s}=1, \cdots, n_{s}, s=1, \cdots, p$ while all other elements of $B$ vanish.

Let a set of $p$-way matrices of order $n$ be given by $B_{1}, \cdots, B_{m}$. The characteristic matrix of $B_{1}, \cdots, B_{m}$ is the matrix $W=\left(\rho_{1} B_{1}+\cdots+\rho_{m} B_{m}\right)$ where $\rho_{1}, \cdots, \rho_{m}$ are parameters.

3. Similar transformations. Non-singular linear transformations on the sets of variables $x_{j_{1}}{ }^{(1)}, \cdots, x_{i_{p}}{ }^{(1)}, j_{1}, \cdots, j_{p}=1, \cdots, n$, of

$$
F=\sum_{i_{1}=\cdots=i_{p}=1}^{n} x_{j_{1}}^{(1)} \cdots x_{i_{p}}^{(p)} \text {, }
$$

which leave $F$ invariant form a similar transformation on $F$. Two bilinear forms and their associated matrices which are equivalent under a similar transformation are similar in the sense of Dickson. $\dagger$ Let matrices $C_{1}, \cdots, C_{p}$ of order $n$ with elements in a field $\phi$ be given by $\left(c_{j_{i_{1}}}^{(1)}\right), \cdots,\left(c_{j_{p_{i}}}^{(p)}\right)$ respectively, where these matrices are associated with the transformations

$$
\begin{aligned}
& x_{j_{1}}^{(1)}=c_{j_{1} i_{1}}^{(1)} y_{i_{1}}^{(1)}, \\
& { }_{j_{2}}^{(2)}=c_{j_{2} i_{2}}^{(2)} y_{i_{2}}^{(2)}, \\
& \cdot \cdot \cdot \cdot \cdot \\
& x_{i_{p}}^{(p)}=c_{j_{p} i_{p}}^{(p)} y_{i_{p}}^{(p)} .
\end{aligned}
$$

Assume that $p \geqq 3$, and that $\left(1_{1}\right),\left(1_{2}\right), \cdots,\left(1_{p}\right)$ leave $F$ invariant, whence

$$
\left(c_{\alpha i_{1}}^{(1)} \cdots c_{\alpha i_{p}}^{(p)}\right)=\delta \text {, }
$$

where $\delta$ is defined on page 422. The layer $\delta_{1}$ of $\delta$ determined by setting $i_{1}=1$ can be written as $\delta_{1}=\Gamma C_{p}$, where

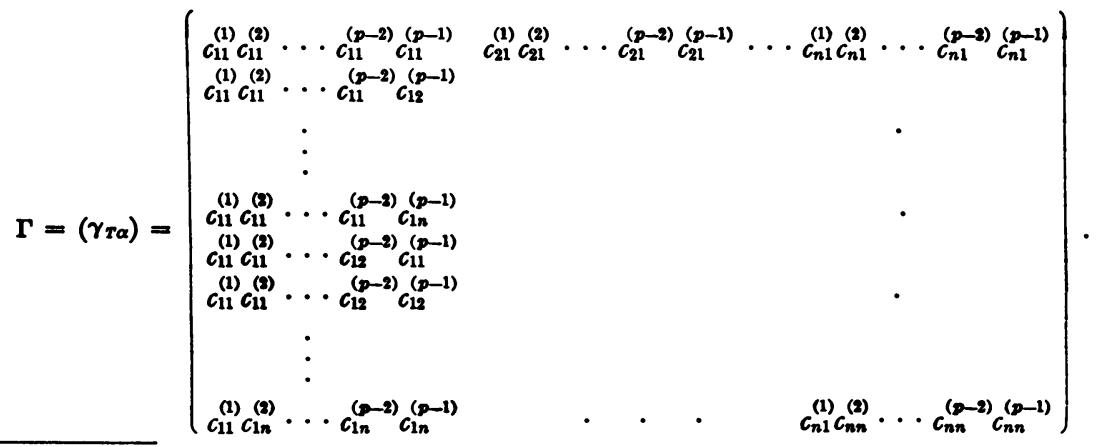

† L. E. Dickson, Modern Algebraic Theories, p. 104. 
$T$ is the partition $i_{2} i_{3} \cdots i_{p-1}$. The second-order minors of the $\xi, \chi$ columns of $\Gamma$ are of the form

$$
M \xi_{x}=c_{\xi_{1}}^{(1)} c_{x 1}^{(1)} K \xi_{x}
$$

where

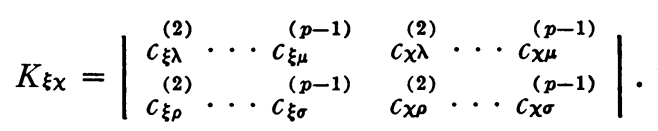

$K_{\xi \chi}$ is a minor of the display $\theta=\left(\theta_{\alpha T}\right)$ of the matrix $M=\left(c_{\alpha i_{2}}^{(2)} \cdots c_{\alpha i_{p-1}}^{(p-1)}\right)$, $\alpha$ not summed. Let a matrix $M^{\prime}$ be given by $\left(C_{i_{2} \alpha_{2}}^{(2)} \cdots C_{i_{p-1} \alpha_{p-1}}^{(p-1)}\right)$, where $\left(C_{i_{2} \alpha_{2}}^{(2)}\right), \cdots,\left(C_{i_{p-1} \alpha_{p-1}}^{(p-1)}\right)$ are the reciprocals of $\left(c_{\alpha_{2}}^{(2)}\right), \cdots,\left(c_{\alpha_{i}}^{(p-1)}\right)$ respectively. Let $\psi=\left(\psi_{T_{\beta}}\right)$ be the 2 -way display of $M^{\prime}$ obtained by letting the partition $T$ be the index of the rows of $\psi$ and $\beta=\alpha_{2} \alpha_{3} \cdots \alpha_{p-1}$ the index of the columns of $\psi$. Evidently

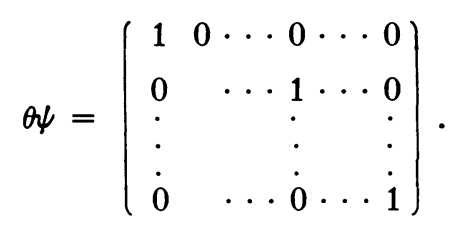

Hence the rank of $\theta$ is $n$.

Since $C_{p}$ is non-singular and the $i_{p}$-rank of $\delta_{1}$ is 1 , the display $\Gamma$ is of rank 1. Hence for $\xi, \chi$ given, the minors $M_{\xi x}$ of $\Gamma$ vanish. Since $\theta$ is of rank $n$ as displayed, the minors $K_{\xi x}$ do not all vanish for given values of $\xi$, $\chi$, whence the products $c_{\xi_{1}}^{(1)} c_{x 1}^{(1)}$ vanish for all values of $\xi, \chi$. Take $c_{11}^{(1)} \neq 0$. Then $c_{x 1}^{(1)}=0$ for all $\chi \neq 1$. Similarly take $c_{i i}^{(1)} \neq 0$ for $i=2, \cdots, n$. Then $c_{\alpha i_{1}}^{(1)}=0$ for $\alpha \neq i_{1}$. This determines a matrix $C_{1}$ which satisfies $(2)$. All other matrices $\left(c_{\alpha i_{1}}^{(1)}\right)$ which satisfy (2) are obtained from $C_{1}$ by arbitrary reordering of the rows and columns of $C_{1}$.

Since $c_{\alpha i_{1}}^{(1)} \cdots c_{\alpha i_{p}}^{(p)}=1$ when $i_{1}=i_{2}=\cdots=i_{p}$, it follows that if $C_{1}$ is a diagonal matrix, $c_{\alpha \alpha}^{(2)}, c_{\alpha \alpha}^{(3)}, \cdots, c_{\alpha \alpha}^{(p)} \neq 0$, and

$$
c_{\alpha \alpha}^{(p)}=\frac{1}{c_{\alpha \alpha}^{(1)} \cdots c_{\alpha \alpha}^{(p-1)}} .
$$

Further since $c_{\alpha i}^{(1)} \cdots c_{\alpha i_{p}}^{(p)}=0$ when $\left(i_{1}, \cdots, i_{p}\right) \neq(\alpha, \cdots, \alpha)$, taking $i_{1}=\cdots=i_{p-1} \neq i_{p}$ we get $c_{\alpha i_{p}}^{(p)}=0$ when $\alpha \neq i_{p}$. Similarly, $c_{\alpha i_{2}}^{(2)}=0$ when $\alpha \neq i_{2}, \cdots, c_{\alpha i_{p-1}}^{(p-1)}=0$ when $\alpha \neq i_{p-1}$.

Evidently all of the solutions of (2) can be obtained from the diagonal 
matrices $C_{1}, \cdots, C_{p}$ as determined above by simultaneous interchanges* of the rows and simultaneous interchanges of the columns of $C_{1}, \cdots, C_{p}$. We have proved

Theorem 1. If $C_{1}, \cdots, C_{p}$ are the $p$ matrices, $p \geqq 3$, of order $n$ associated with a similar transformation in a field $\phi$, then $C_{1}, \cdots, C_{p}$ are diagonal matrices satisfying the condition $C_{p}=C_{1}^{-1} \cdots C_{p-1}^{-1}$ or matrices obtained from these diagonal matrices by simultaneous interchanges of the rows and simultaneous interchanges of the columns of these matrices.

In the case $p=2$, as is well known, the matrices $C_{1}, C_{2}$ associated with a similar transformation satisfy the property $C_{2}=C_{1}^{\prime}{ }^{-1}, C_{1}{ }^{\prime}$ being the transpose of $C_{1}$, but are not necessarily diagonal. We have here a case where the theory for $p$-way matrices, $p \geqq 3$, is much simpler than that for 2 -way matrices.

By Theorem 1 the canonical $\dagger$ pairs of $p$-way matrices, where one of the matrices is a $\delta$-matrix, can be written down.

An effect of a similar transformation on a given matrix is stated in

THEOREM 2. Under a similar transformation in a field $\phi$ on the indices $i_{1}, \cdots, i_{p}$ the $\left(i_{1}, \cdots, i_{p}\right)$ diagonal elements of a p-way matrix $A=\left(a_{i_{1}} \ldots i_{p}\right)$, $p \geqq 3$, are at most rearranged.

Evidently, $a_{\alpha_{1} \cdots \alpha_{p}} c_{\alpha_{1} i}^{(1)} \cdots c_{\alpha_{p} i}^{(p)}=a_{\alpha} \cdots \alpha c_{\alpha i}^{(1)} \cdots c_{\alpha i}^{(1)}$, where $\alpha$ has some value between 1 and $n . \alpha$ in the above relation is assumed not summed.

4. Preliminary theorems. We shall prove

THEOREM 3. If a p-way matrix $A=\left(a_{i_{1}} \ldots i_{p}\right), p \geqq 3$, with elements in a field $\phi$, is equivalent under non-singular linear transformations in $\phi$ to a $\delta$-matrix on $\left(i_{1}, \cdots, i_{p}\right)$, the characteristic matrix $M=\left(\rho_{1} a_{1 i_{2}} \cdots i_{p}+\cdots+\rho_{n} a_{n i_{2}} \cdots i_{p}\right)$ can be chosen non-singular for at least one set of values of $\rho_{1}, \cdots, \rho_{n}$ in $\phi$.

Let $\delta=\left(\delta_{j_{1} \cdots j_{p}}\right)$ represent a $\delta$-matrix on $\left(j_{1} \cdots j_{p}\right)$, and let $\delta_{1}=\left(\delta_{1 j_{2}} \cdots j_{p}\right)$, $\delta_{2}=\left(\delta_{2 j_{2}} \cdots j_{p}\right), \cdots, \delta_{n}=\left(\delta_{n j_{2}} \cdots j_{p}\right)$. Let the characteristic matrix $\left(\rho_{1} \delta_{1}+\cdots\right.$ $\left.+\rho_{n} \delta_{n}\right)$ of $\delta_{1}, \cdots, \delta_{n}$ be given by $W=\left(w_{j_{2}} \cdots j_{p}\right)$. The $i_{1}$-layers $U_{1}, \cdots, U_{n}$ of the matrix $L=\left(c_{j_{1} i_{1}}^{(1)} \delta_{j_{1}} \cdots_{j_{p}}\right)$ obtained after the non-singular linear transformation $\left(1_{1}\right)$ on $x_{j_{1}}^{(1)}$ of

$$
F=\sum_{j_{1}=\cdots=j_{p}=1}^{n} x_{j_{1}}^{(1)} \cdots x_{i_{p}}^{(p)}
$$

are related to $\delta_{1}, \cdots, \delta_{n}$ by the relations

\footnotetext{
* By a simultaneous interchange of the $i$ and $j$ rows of $C_{1}, \cdots, C_{p}$ is meant the interchange of the $i$ and $j$ rows of $C_{1}, \cdots$, the interchange of the $i$ and $j$ rows of $C_{p}$.

$\dagger$ "Canonical" is used in the same sense as in the ordinary theory of bilinear forms and 2-way matrices. See Dickson, op. cit., p. 89 ff.
} 


$$
\begin{gathered}
U_{1}=c_{11}^{(1)} \delta_{1}+\cdots+c_{n 1}^{(1)} \delta_{n}, \\
\cdots \cdot \cdots+\cdots \\
U_{n}=c_{1 n}^{(1)} \delta_{1}+\cdots+c_{n n}^{(1)} \delta_{n}
\end{gathered}
$$

The characteristic matrix $W^{\prime}$ of $U_{1}, \cdots, U_{n}$ is given by $W^{\prime}=\left(\sigma_{1} U_{1}+\cdots\right.$ $\left.+\sigma_{n} U_{n}\right)$. $W^{\prime}$ can be obtained from $W$ by the non-singular linear transformation on the $\rho$ 's given by

$$
\begin{aligned}
& \rho_{1}=c_{11}^{(1)} \sigma_{1}+\cdots+c_{1 n}^{(1)} \sigma_{n}, \\
& \cdot \cdot \cdot \cdot \cdot \cdot \cdot \cdot \\
& \rho_{n}=c_{n 1}^{(1)} \sigma_{1}+\cdots+c_{n n}^{(1)} \sigma_{n} .
\end{aligned}
$$

If $\rho_{1}=\cdots=\rho_{n}=1$, then $W$ is the non-singular $\delta$-matrix on $\left(j_{2}, \cdots, j_{p}\right)$. By equations (3), $\sigma_{1}, \cdots, \sigma_{n}$ can be so chosen that $W^{\prime}$ is non-singular.

Non-singular linear transformations $\left(1_{2}\right), \cdots,\left(1_{p}\right)$ on $x_{j_{2}}^{(2)}, \cdots, x_{j_{p}}^{(p)}$ associated with $L$ correspond to the transition from $W^{\prime}=\left(W_{j_{2}}^{\prime} \ldots j_{p}\right)$ to $W^{\prime \prime}$ $=\left(W_{j_{2}} \cdots j_{j} c_{j_{2} i_{2}}^{(2)} \cdots c_{j_{p} i_{p}}^{(p)}\right)$. If $\sigma_{1}, \cdots, \sigma_{n}$ are so chosen that $W^{\prime}=\left(e_{\xi_{j_{2}}}^{(2)} \cdots e_{\xi_{j}}^{(p)}\right)$, where $\left(e_{\xi_{2}}^{(2)}\right), \cdots,\left(e_{\xi_{p}}^{(p)}\right)$ are non-singular matrices, then

$$
W^{\prime \prime}=\left(e_{\xi_{2}}^{(2)} c_{j_{2} i_{2}}^{(2)} \cdots e_{\xi_{j} j_{p}}^{(p)} c_{j_{p_{p}}}^{(p)}\right) \text {, }
$$

which is obviously non-singular. This completes the proof of the theorem.

Theorem 3 gives a necessary condition for the non-singularity of a given matrix. If $p=3$ it is very easy to test a given matrix by means of the theorem. For $p>3$ no simple general method has been found for applying it.

Let $T=\left(T_{1}, \cdots, T_{m}\right)$ denote a set of 2-way matrices of order $n$ with elements in a field $\phi$. If the set $T$ is to be equivalent to a set of diagonal matrices under similar transformation* in $\phi$ it is necessary that $T_{1}$ be equivalent under similar transformation in $\phi$ to a matrix of the form

$$
D=\left(\begin{array}{ccc}
r_{1} I_{1} & & \\
& \cdot & 0 \\
& 0 & \cdot \\
& & r_{\mu} I_{\mu}
\end{array}\right),
$$

where $r_{1}, \cdots, r_{\mu}$ are mutually distinct scalars, and $I_{1}, \cdots, I_{\mu}$ are Kronecker deltas. The condition for such equivalence is given in the

LEMMA. A 2-way matrix $T_{1}$ is equivalent under a similar transformation in $\phi$ to a matrix of type $D$ if and only if the invariant factors of $\left(T_{1}-\lambda I\right)$, where $I$ is a Kronecker delta, split up into distinct linear factors in $\phi$.

* For a treatment of the equivalence of 2-way matrices under similar transformations see L. E. Dickson, op. cit., p. $89 \mathrm{ff}$. 
Let the set $T$ now be reduced to $D, T_{2}^{\prime}, \cdots, T_{m}^{\prime}$ under similar transformation in $\phi$, and let

$$
T_{s}^{\prime}=\left(\begin{array}{cccc}
T_{11}^{s} & T_{12}^{s} & \cdots & T_{1 \mu}^{s} \\
T_{21}^{s} & \cdot & \cdots & : \\
T_{\mu 1}^{s} & \cdot & \cdots & T_{\mu \mu}^{s}
\end{array}\right)
$$$$
(s=2, \cdots, m),
$$

where $T_{i i}^{s}$ is of the same order as $I_{i}$ for $i=1, \cdots, \mu$. The most general matrix $X$ which satisfies the relation $X D X^{-1}=D$ is of the form

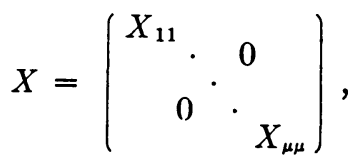

where $X_{\sigma \sigma}$ is of the same order as $I_{\sigma}$ for $\sigma=1, \cdots, \mu$. Under similar transformation with $X, X^{-1}$ the matrix $T_{s}^{\prime}$ goes into

$$
T_{s}^{\prime \prime}=\left(\begin{array}{cccc}
X_{11} T_{11}^{s} X_{11}^{-1} & X_{11} T_{12} X_{22}^{-1} & \cdots & X_{11} T_{1 \mu}^{s} X_{\mu \mu}^{-1} \\
X_{22} T_{21}^{s} X_{11}^{-1} & \vdots & \cdots & \vdots \\
\vdots & \vdots & \cdots & \vdots \\
X_{\mu \mu} T_{\mu 1}^{s} X_{11}^{-1} & \cdot & \cdots & X_{\mu \mu} T_{\mu \mu}^{s} X_{\mu \mu}^{-1}
\end{array}\right)
$$

for $s=2, \cdots, m$. If $T_{s}^{\prime \prime}$ are diagonal matrices, $X_{\alpha \alpha} T_{\alpha \beta}^{z} X_{\beta \beta}^{-1}=0$ for all $s$ and $\alpha \neq \beta$, whence $T_{\alpha \beta}^{s}=0$ for all $s$ and $\alpha \neq \beta$. Further, for every $\sigma$, the matrices $X_{\sigma \sigma} T_{\sigma \sigma}^{2} X_{\sigma \sigma}^{-1}, \cdots, X_{\sigma \sigma} T_{\sigma \sigma}^{m} X_{\sigma \sigma}^{-1}$ must be diagonal matrices. We have proved

THEOREM 4. Let $T_{1}$ of the set $T=\left(T_{1}, \cdots, T_{m}\right)$ of 2-way matrices of order $n$ satisfy the Lemma. The set $T$ is equivalent under similar transformations in $\phi$ to a set of diagonal matrices if and only if $T_{\alpha \beta}^{s}=0$ for $\alpha \neq \beta, \alpha \beta=1, \cdots, \mu$, $s=2, \cdots, m$, and the set $\Sigma_{\sigma}=\left(T_{\sigma \sigma}^{2}, \cdots, T_{\sigma \sigma}^{m}\right)$ is equivalent under similar transformation in $\phi$ to a set of diagonal matrices for every $\sigma$ for which the matrices in $\Sigma_{\sigma}$ are of order greater than 1.

The analogue of Theorem 4 for $p$-way matrices, $p \geqq 3$, is given in

THEOREM 5. If $p \geqq 3$, and the $p$-way matrices $T_{1}, \cdots, T_{m}$ of order $n$ with elements in a given field $\phi$ are equivalent under similar transformation in $\phi$ to a set of diagonal matrices, the matrices $T_{1}, \cdots, T_{m}$ are diagonal matrices.

By Theorem 2 the $\left(i_{1}, \cdots, i_{p}\right)$ diagonal elements of a matrix $T_{\sigma}=\left(t_{i_{1}}^{\sigma} \cdots i_{p}\right)$ are at most rearranged under similar transformation on $i_{1}, \cdots, i_{p}$.

Theorem 5 can be used to test the equivalence of a set $T=\left(T_{1}, \cdots, T_{m}\right)$ of $p$-way matrices, $p \geqq 3$, of order $n$, where $T_{1}$ is non-singular, to a set of 
diagonal matrices. Under reduction of $T_{1}$ to $\delta$, where $\delta$ is a $p$-way $\delta$-matrix of order $n$, the matrices $T_{2}, \cdots, T_{m}$ go into a set $\Sigma=\left(T_{21}, \cdots, T_{m 1}\right)$. It is evident that the set $T$ is equivalent to a set of diagonal matrices if and only if the set $\Sigma$ is equivalent to a set of diagonal matrices under similar transformation.

5. Necessary and sufficient conditions for a matrix to be non-singular. We have the following factorization property of non-singular matrices.

TheOREM 6. $A$ p-way matrix $A=\left(a_{i_{1}} \ldots i_{p}\right), p \geqq 3$, is non-singular if and only if $A$ can be factored into the form $\left(c_{\alpha i_{1}}^{(1)} \cdots c_{\alpha i_{p}}^{(p)}\right)$, where $\left(c_{\alpha i_{1}}^{(1)}\right), \cdots,\left(c_{\alpha i_{p}}^{(p)}\right)$ are non-singular.

The $\delta$-matrix $\left(\delta_{j_{1}} \ldots j_{p}\right)$ on $\left(j_{1}, \cdots, j_{p}\right)$ can be written as $\left(\delta_{\alpha j_{1}} \ldots \delta_{\alpha j_{p}}\right)$, where $\left(\delta_{\alpha j_{1}}\right), \cdots,\left(\delta_{\alpha j_{p}}\right)$ are Kronecker deltas. If $A$ is non-singular, we have

$$
\left(a_{i_{1}} \cdots i_{p}\right)=\left(\delta_{j_{1} \cdots j_{p}} c_{j_{1} i_{1}}^{(1)} \cdots c_{i_{p} i_{p}}^{(p)}\right)=\left(\delta_{\alpha j_{1}} c_{j_{1} i_{1}}^{(1)} \cdots \delta_{\alpha j_{p}}^{\left(p j_{p} i_{p}\right.}\right)=\left(c_{\alpha i_{1}}^{(p)} \cdots c_{\alpha i_{p}}^{(p)}\right),
$$

where $\left(c_{\alpha i_{1}}^{(1)}\right), \cdots,\left(c_{\alpha i_{p}}^{(p)}\right)$ are non-singular 2-way matrices.

Every matrix which can be written in the form

$$
\left(c_{\alpha i_{1}}^{(1)} \cdots c_{\alpha i_{p}}^{(p)}\right) \quad(\alpha=1, \cdots, n),
$$

where the rank of $\left(c_{\alpha i_{1}}^{(1)}\right)$ is $m$ and the ranks of $\left(c_{\alpha i_{2}}^{(2)}\right), \cdots,\left(c_{\alpha i_{p}}^{(p)}\right)$ are all equal to $n$, can (regardless of the ranges of $i_{1}, \cdots, i_{p}$ ) be reduced under elementary transformations* to

$$
N=\left(c_{\alpha i_{1}}^{(1)} \cdots c_{\alpha i_{p}}^{(p)}\right) \quad\left(i_{1}=1, \cdots, m ; \alpha, i_{2}, \cdots, i_{p}=1, \cdots, n\right),
$$

or $N$ bordered by zeros. Our theorems, which will be stated for $N$ instead of (4), will therefore hold for more general cases.

Let $E$ denote the matrix $\left(e_{i_{1}} \ldots i_{p}\right) ; i_{1}=1, \cdots, m ; i_{2}, \cdots, i_{p}=1, \cdots, n$. We shall now prove

THEOREM 7. The matrix $E$ with elements in a field $\phi$ is factorable into a matrix of type $N$ with elements in $\phi$ if and only if $E$ is non-singular on $i_{1}$, and the $i_{1}$-layers of $E$ are equivalent in $\phi$ to a set of diagonal matrices.

The $i_{1}$-layers of $E$ must be linearly independent since the $i_{1}$-rank of $N$ is $m$ and this rank is invariant under non-singular linear transformations.

Let $\left(C_{i_{2} \alpha_{2}}^{(2)}\right), \cdots,\left(C_{i_{p} \alpha_{p}}^{(p)}\right)$ be the reciprocals of $\left(c_{\alpha_{1}}^{(2)}\right), \cdots,\left(c_{\alpha i_{p}}^{(p)}\right)$ respectively. If $E=N$, then

$$
\left(e_{i_{1} \cdots i_{p}} C_{i_{2} \alpha_{2}}^{(2)} \cdots C_{i_{p} \alpha_{p}}^{(p)}\right)=\left(c_{\alpha i_{1} \delta_{\alpha \alpha_{2}}}^{(1)} \cdots \delta_{\alpha \alpha_{p}}\right),
$$

* For a discussion of elementary transformations see Bôcher, Introduction to Higher Algebra, p. 55. Elementary transformations leave the factorization property (4) invariant. 
where $\left(\delta_{\alpha \alpha_{2}}\right), \cdots,\left(\delta_{\alpha \alpha_{p}}\right)$ are Kronecker deltas of order $n$. Equation (5) is equivalent to the set

$$
\left(e_{1 i_{2} \cdots i_{p}} C_{i_{2} \alpha_{2}}^{(2)} \cdots C_{i_{p} \alpha_{p}}^{(p)}\right)=\left(c_{\alpha i_{1} \delta_{\alpha \alpha_{2}}}^{(1)} \cdots \delta_{\alpha \alpha_{p}}\right),
$$

$$
\left(e_{m i_{2} \cdots i_{p}} C_{i_{2} \alpha_{2}}^{(2)} \cdots C_{i_{p} \alpha_{p}}^{(p)}\right)=\left(c_{\alpha m}^{(1)} \delta_{\alpha \alpha_{2}} \cdots \delta_{\alpha \alpha_{p}}\right) .
$$

The matrices $\left(c_{\alpha 1}^{(1)} \delta_{\alpha \alpha_{2}} \cdots \delta_{\alpha \alpha_{p}}\right), \cdots,\left(c_{\alpha m}^{(1)} \delta_{\alpha \alpha_{2}} \cdots \delta_{\alpha \alpha_{p}}\right)$ are diagonal matrices.

We have proved that if $E$ is factorable into a matrix $N$, then the $i_{1}$-layers of $E$ are linearly independent, and equivalent under non-singular linear transformations in $\phi$ to a set of diagonal matrices; the converse is simply proved.

We have now determined enough conditions to test the non-singularity of a given matrix $A=\left(a_{i_{1}} \cdots i_{p}\right), i_{1}, \cdots, i_{p}=1, \cdots, n$. The procedure of this test is as follows. Determine whether or not the necessary condition of Theorem 7 concerning the non-singularity of $A$ on $i_{1}$ is satisfied. If $A$ is non-singular on $i_{1}$, determine (if possible) whether or not the necessary condition of Theorem 3 is satisfied. If so, choose the matrix $M$ mentioned in Theorem 3 so that it is non-singular. Let the $\rho$ 's and the $i_{1}$-layers of $A$ be ordered so that $\rho_{1} \neq 0$. Let $A_{1}, \cdots, A_{n}$ designate the $i_{1}$-layers of $A$. Determine by Theorems 4 and 5 whether or not the set $M, A_{2}, \cdots, A_{n}$ is equivalent to a set of diagonal matrices. If not, $A$ is singular. ${ }^{*}$ If the contrary is the case, and $p=3$ (similarly for $p \geqq 4$ ), then there exist non-singular matrices $X, Y$ such that

$$
X \rho_{i} A_{i} Y=D_{1}, X A_{2} Y=D_{2}, \cdots, X A_{n} Y=D_{n},
$$

where $D_{1}, \cdots, D_{n}$ are diagonal matrices. Multiplying the last $(n-1)$ equations by $\rho_{2}, \cdots, \rho_{n}$ respectively and subtracting the resulting equations from the first, we obtain $X \rho_{1} A_{1} Y=D_{1}-\sum_{i=2}^{n} \rho_{i} D_{i}$. The matrix on the right is a diagonal matrix, from which it follows that the set $A_{1}, \cdots, A_{n}$ is equivalent to a set of diagonal matrices. Theorem 7 is now satisfied, and $A$ is non-singular. In certain situations the non-singularity of $A$ is at once evident from Theorem 6.

Let $A_{1}, \cdots, A_{n}$ be the $i_{1}$-layers of $A=\left(a_{i_{1} \cdots i_{p}}\right)$ with elements in a field $\phi$ and $p \geqq 4$, and let $\rho_{1}, \cdots, \rho_{n}$ be chosen so that the characteristic matrix $M=\left(\sum_{i=1}^{n} \rho_{i} A_{i}\right)$ is non-singular for $\rho_{1} \neq 0$. Let the transformations which reduce $M$ to a $\delta$-matrix reduce $A_{2}, \cdots, A_{n}$ to $A_{2}^{\prime}, \cdots, A_{n}^{\prime}$. Theorems 5 and 7 imply

THEOREM 8. The matrix $A=\left(a_{i_{1}} \cdots i_{p}\right), p \geqq 4$, is non-singular if and only if $A$ is non-singular on $i_{1}$, and the matrices $A_{2}^{\prime}, \cdots, A_{n}^{\prime}$ are diagonal matrices.

\footnotetext{
* A matrix not non-singular is said to be singular.
} 
If $A=\left(a_{i_{1} i_{2} i_{3}}\right)$ is a 3-way non-singular matrix of order $n$ and $A_{1}, \cdots, A_{n}$ are the $i_{1}$-layers of $A$, by Theorem 7 there exist non-singular matrices $X, Y$ such that

$$
X A_{i} Y=Q_{i} \quad(i=1, \cdots, n),
$$

where $Q_{i}$ are diagonal matrices. The matrices $X, Y$ can be obtained from Theorem 4 and the theory of bilinear forms. Arrange the matrices $Q_{i}$ to form the rows of a 2-way matrix $P$. Since the $i_{1}$-rank of $A$ is $n$, there exists a nonsingular minor $V$ of $P$. The matrix $V^{-1} P$ is a 2-way display of a matrix $\delta$, where $\delta$ is a 3 -way $\delta$-matrix of order $n$. The matrices $V^{\prime-1}, X^{\prime}, Y$ (the primes here denote transpose) are hence matrices which reduce $A$ to $\delta$ under transformation on $i_{1}, i_{2}, i_{3}$ respectively. The matrices of reduction from a $p$-way non-singular matrix, $p \geqq 4$, to a $\delta$-matrix are obtained similarly.

We define the factorization rank of a matrix $A=\left(a_{i_{1}} \ldots i_{p}\right)$ to be the minimum value of $\epsilon$ for which the matrix $A$ can be written in the form $\left(\sum_{\alpha=1}^{e} c_{\alpha i_{1}}^{(1)} \cdots c_{\alpha i_{p}}^{(p)}\right)$, where $\left(c_{\alpha i_{1}}^{(1)}\right), \cdots,\left(c_{\alpha i_{p}}^{(p)}\right)$ are 2 -way matrices. This rank is invariant under non-singular linear transformations. The factorization rank of a matrix $\left(\sum_{\alpha=1}^{e} c_{\alpha i_{1}}^{(1)} \cdots c_{\alpha i_{p}}^{(p)}\right)$ is $n$ if all of the matrices $\left(c_{\alpha i_{1}}^{(1)}\right), \cdots,\left(c_{\alpha i_{p}}^{(p)}\right)$ are non-singular.

In another paper* the author has defined certain ranks of a $p$-way matrix which are invariant under non-singular linear transformations. The following theorem is easily proved.

Theorem 9. A p-way matrix $A$ of order $n$ is non-singular if and only if all of its invariant ranks are equal to $n$.

6. Note on invariant factors. The matrix $W=\left(\sum_{i=1}^{m} \rho_{i} B_{i}\right)$ used in $\S 2$ suggests the following generalization of ordinary invariant factor theory. Let $B_{i}, i=1, \cdots, m$, be square matrices of order $n$. Let $G_{t}$ be the greatest common divisor of the minors of $W$ of the $t$ th order, and let $G_{0}=1$. We define the $t$ th invariant factor of $W$ to be the quotient $G_{t} / G_{t-1}$. It is determined up to a constant factor. It is assumed in factoring the minors of $M$ to obtain the $G_{t}$, $t=0,1, \cdots, n$, that the factorization is performed in a given field. $\dagger$ Now when $B_{1}, \cdots, B_{m}$ are multiplied. by non-singular matrices the quotients $G_{t} / G_{t-1}$ are invariant. If

$$
\begin{aligned}
& B_{1}^{\prime}=a_{11} B_{1}+\cdots+a_{1 m} B_{m}, \\
& \cdot \cdot \cdot \cdot \cdot \cdot \cdot \cdot \cdot \\
& B_{m}^{\prime}=a_{1 m} B_{1}+\cdots+a_{m m} B_{m},
\end{aligned}
$$

* Composition and rank of $n$-way matrices and multilinear forms, Annals of Mathematics, vol. 35 (1934), pp. 625, 633, 634.

$\dagger$ It is to be noted that for $m \geqq 3$ a minor of $W$ cannot always be factored into distinct linear factors in a field. To obtain linear factors it is necessary in general to use the quasi-field of quaternions and other generalizations of fields. 
and the matrix of coefficients is non-singular, then the invariant factors of $W^{\prime}=\left(\alpha_{1} B_{1}^{\prime}+\cdots+\alpha_{m} B_{m}^{\prime}\right)$ can be obtained from the invariant factors of $W$ by the transformations

$$
\begin{aligned}
& \rho_{1}=a_{11} \alpha_{1}+\cdots+a_{m 1} \alpha_{m}, \\
& \cdot . \cdot \cdots+a_{m m} \alpha_{m} .
\end{aligned}
$$

Hence powers of terms occurring in invariant factors go into like powers under the transformation from the set $B_{1}, \cdots, B_{m}$ to $B_{1}^{\prime}, \cdots, B_{m}{ }^{\prime}$.

The determinant of $W$ in the proof of Theorem 3 where $p=3$ is the only invariant factor of $W$ distinct from unity. This determinant factors into distinct linear factors in any field $\phi$. It follows that if $\Delta$ is the determinant of the characteristic matrix of the $i$-layers of $R=\left(r_{i j k}\right)$ and $R$ is equivalent in $\phi$ to a $\delta$-matrix on $(i, j, k)$, then $\Delta$ factors into distinct linear factors in $\phi$, and is the only invariant factor distinct from a constant.

An exact generalization of the theory of this section holds for $p$-way matrices $p \geqq 3$, where the invariant factors are defined in terms of space determinants.

Chapter II. Factorization of $p$-Way matrices into a product OF 2-WAY MATRICES ONE OF WHICH IS SINGULAR

7. Introduction. By Theorem 7, a matrix $A=\left(a_{i_{1}} \ldots i_{p}\right) ; i_{1}=1, \cdots, m$; $i_{2}, \cdots, i_{p}=1, \cdots, n$, can be factored into a matrix $A=\left(c_{\alpha i_{1}}^{(1)} \cdots c_{\alpha i_{p}}^{(p)}\right)$; $\alpha=1, \cdots, n$, where $\left(c_{\alpha i_{1}}^{(1)}\right), \cdots,\left(c_{\alpha i_{p}}^{(p)}\right)$ are non-singular on $i_{1}, \cdots, i_{p}$ respec- $^{-}$ tively, if and only if the $i_{1}$-layers of $A_{1}, \cdots, A_{m}$ of $A$ are linearly independent, and are simultaneously equivalent to a set of diagonal matrices. To complete the treatment of factorizations of the above type where $\left(c_{\alpha i_{1}}^{(1)}\right)$ is singular we must obtain necessary and sufficient conditions for the equivalence of $A_{1}, \cdots, A_{m}$ to a set of diagonal matrices. We do not assume as in the treatment of a non-singular matrix $A$ that there exist values of $\rho_{1}, \cdots, \rho_{m}$ not all zero such that $\rho_{1} A_{1}+\cdots+\rho_{m} A_{m}$ is non-singular.

Using an essentially different technique we derive first (Theorem 10) the canonical diagonal 2-way matrices $C_{1}, \cdots, C_{q}$ to which diagonal matrices $E_{1}, \cdots, E_{q}$ are equivalent under non-singular linear transformations, and then obtain necessary and sufficient conditions (Theorems 12,13) for the equivalence of a set of 2-way matrices $C_{1}, \cdots, C_{i}, F_{i+1}$ to a set of diagonal matrices.

To test the equivalence of a set $S=\left(A_{1}, \cdots, A_{m}\right)$ of 2-way matrices to a set of diagonal matrices, reduce $A_{1}$ to a canonical diagonal matrix $C_{1}$. If this is not possible (this is very easy to determine), it follows that the set $S$ 
is not equivalent to a set of diagonal matrices. If $A_{1}$ is equivalent to a matrix $C_{1}$, reduce $A_{1}$ to $C_{1}$. The remaining matrices of $S$ are simultaneously transformed into a set $A_{2}^{\prime}, \cdots, A_{m}^{\prime}$ respectively. Apply Theorems 12 and 13 to determine whether or not the pair $C_{1}, A_{2}^{\prime}$ is equivalent to a pair of canonical diagonal matrices $C_{1}, C_{2}$. If not, the set $S$ is not equivalent to a set of diagonal matrices. If on the other hand the pair $C_{1}, A_{2}^{\prime}$ is equivalent to a pair $C_{1}, C_{2}$, reduce $C_{1}, A_{2}^{\prime}$ to $C_{1}, C_{2}$. The remaining matrices in the set $S$ are then transformed into a set $A_{3}^{\prime \prime}, \cdots, A_{m}{ }^{\prime \prime}$. Now apply Theorems 12 and 13 to $C_{1}, C_{2}, A_{3}^{\prime \prime}$. Continue this process until one finally arrives at a set of canonical diagonal matrices $C_{1}, \cdots, C_{m}$ to which the set $S$ is equivalent.

8. Canonical diagonal 2-way matrices. Adopting the notation used by J. Williamson in a recent paper* we shall write the "diagonal block matrix"

$$
C=\left(\begin{array}{ccc}
C_{1} & & \\
\cdot & 0 \\
0 & \cdot \\
& & C_{s}
\end{array}\right),
$$

where $C_{1}, \cdots, C_{s}$ are square minors of $C$, in the form

$$
C=\left[C_{1}, \cdots, C_{s}\right] .
$$

Let the letters $I, \rho$ with superscripts and subscripts denote a Kronecker delta, and a parameter respectively. We shall prove

THEOREM 10. Let $S=\left(E_{1}, \cdots, E_{q}\right)$ denote a set of diagonal 2-way matrices of order $n$ with elements in a given field $\phi$. The set $S$ is equivalent in $\phi$ to a set $S^{\prime}=\left(C_{1}, \cdots, C_{q}\right)$, where

$$
\begin{aligned}
& C_{1}=\left[I_{1}^{1}, 0\right], \\
& C_{2}=\left[S_{1}^{1}, S_{2}^{1}\right],
\end{aligned}
$$

and

$$
\begin{aligned}
& S_{1}^{1}=\left[\rho_{1}^{2} I_{1}^{2}, \cdots, \rho_{t}^{2} I_{t}^{2}\right], \\
& S_{2}^{1}=\left[I_{t+1}^{2}, 0\right] ;
\end{aligned}
$$

the $\rho_{1}{ }^{2}, \cdots, \rho_{t}{ }^{2}$ are all distinct, and the matrix $S_{1}{ }^{1}$ is of the same order as $I_{1}{ }^{1}$; in general any pair in the set $S^{\prime}$ is of the form

$$
\begin{aligned}
& \left(7_{i}\right) \quad C_{i}=\left[\rho_{1}^{i} I_{1}^{i}, \cdots, \rho_{s(i)-1}^{i} I_{s(i)-1}^{i}, 0 \cdot I_{s(i)}^{i}\right], \\
& \left(7_{i+1}\right) \quad C_{i+1}=\left[S_{1}^{i}, \cdots, S_{s(i)}^{i}\right],
\end{aligned}
$$

where

* Simultaneous reduction of two matrices to triangle form, American Journal of Mathematics, vol. 57 (1935), p. 282. 


$$
\begin{aligned}
& S_{1}^{i}=\left[\rho_{1}^{i+1} I_{1}^{i+1}, \cdots, \rho_{m_{1}}^{i+1} I_{m_{1}}^{i+1}\right], \\
& S_{2}^{i}=\left[\rho_{m_{1}+1}^{i+1} I_{m_{1}+1}^{i+1}, \cdots, \rho_{m_{1}+m_{2}}^{i+1} I_{m_{1}+m_{2}}^{i+1}\right]
\end{aligned}
$$

$\left(9_{s(i)-1}\right) S_{s(i)-1}^{i}=\left[\rho_{m_{1}+\cdots+m_{s(i)-2+1}}^{i+1} I_{m_{1}+\cdots+m_{s(i)-2+1}}^{i+1}, \cdots, \rho_{m_{1}+\cdots+m_{s}(i)-1}^{i+1} I_{m_{1}+\cdots+m_{s(i)-1}}^{i+1}\right]$,

$\left(9_{s(i)}\right) \quad S_{s(i)}^{i}=\left[I_{m_{1}+\cdots+m_{s(i)-1}+1}^{i+1}, 0\right]$,

and where the $\rho$ 's in each matrix $S_{1}^{i}, \ldots, S_{s(i)-1}^{i}$ are distinct, and the orders of the matrices $S_{1}^{i}, \cdots, S_{s(i)}^{i}$ are equal to the orders of $I_{1}^{i}, \cdots, I_{s(i)}^{i}$ respectively.

At the same time we shall prove

THEOREM 11. The matrices $X, Y$ which satisfy the relations

$$
X C_{1} Y=C_{1}, \cdots, X C_{i} Y=C_{i}
$$

are of the form

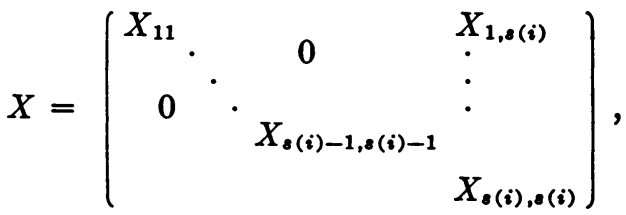

$$
\begin{aligned}
& Y=\left(\begin{array}{cccc}
X_{11}^{-1} & \multicolumn{2}{c}{0} \\
0 & \ddots & \multicolumn{1}{c}{} \\
& X_{8(i)-1,8(i)-1}^{-1} & \\
Y_{8(i), 1} & \cdots & & Y_{s(i), 8(i)}
\end{array}\right),
\end{aligned}
$$

where $X_{11}, \cdots, X_{s(i), s(i)}$ are of the same orders as $I_{1}^{i}, \cdots, I_{s(i)}^{i}$ respectively.

If $E_{1}$ is of rank $r, E_{1}$ is obviously equivalent to $C_{1}$, where $I_{1}^{1}$ is of rank $r$. It is readily verified that if $X C_{1} Y=C_{1}$, then

$$
X=\left(\begin{array}{cc}
X_{11} & X_{12} \\
0 & X_{22}
\end{array}\right), \quad Y=\left(\begin{array}{cc}
X_{11}^{-1} & 0 \\
Y_{21} & Y_{22}
\end{array}\right),
$$

where $X_{11}$ is a minor of order $r$.

Assume now that $E_{1}, \cdots, E_{i}$ have been reduced to canonical forms $C_{1}, \cdots, C_{i}$ and that $X, Y$ are as given in (10). Let $E_{i+1}$ be denoted by

$$
\left[P_{i}^{i+1}, \cdots, P_{s(i)}^{i+1}\right],
$$

where the minors $P_{1}^{i+1}, \cdots, P_{s(i)}^{i+1}$ are of the same orders as $I_{1}^{i}, \cdots, I_{s(i)}^{i}$. Let $X_{1,8(i)}, \cdots, X_{s(i)-1,8(i)}, Y_{s(i), 1}, \cdots, Y_{s(i), 8(i)-1}$ be set equal to zero. Then 


$$
\begin{array}{r}
X E_{i+1} Y=\left[X_{11} P_{1}^{i+1} X_{11}^{-1}, \cdots, X_{s(i)-1,(i)-1} P_{s(i)-1}^{i+1} X_{s(i)-1, s(i)-1}^{-1},\right. \\
\left.X_{s(i), s(i)} P_{s(i)}^{i+1} Y_{s(i), 8(i)}\right] .
\end{array}
$$

Choose the non-zero minors of $X, Y$ so that

$$
X_{11} P_{1}^{i+1} X_{11}^{-1}, \cdots, X_{s(i)-1,8(i)-1} P_{s(i)-1}^{i+1} X_{8(i)-1, s(i)-1}^{-1}
$$

are in the classical canonical forms ${ }^{*} S_{1}^{i}, \cdots, S_{s(i)-1}^{i}$, and choose $X_{s(i), s(i)}$, $Y_{s(i), 8(i)}$ so that $X_{s(i), o(i)} P_{s(i)}^{i+1} Y_{s(i), s(i)}$ is a Kronecker delta bordered by zeros. The matrix $E_{i+1}$ has now been brought into a canonical form of type $C_{i+1}$.

It is readily verified that the matrices $X, Y$ of (10) which satisfy the relation

$$
X C_{i+1} Y=C_{i+1}
$$

are of the form

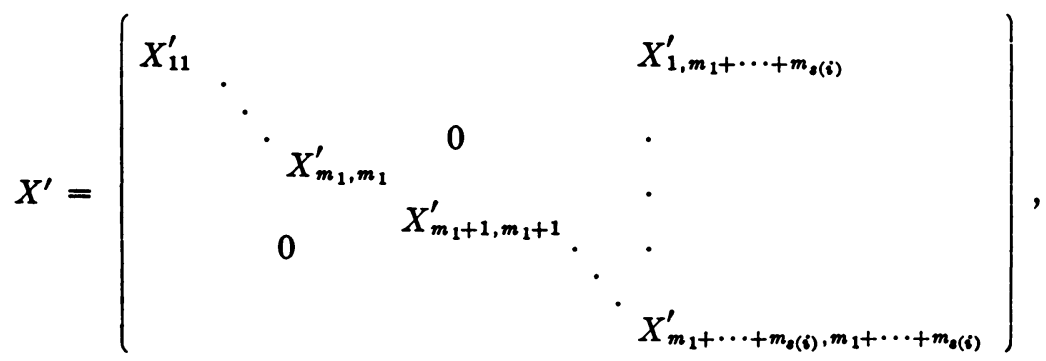

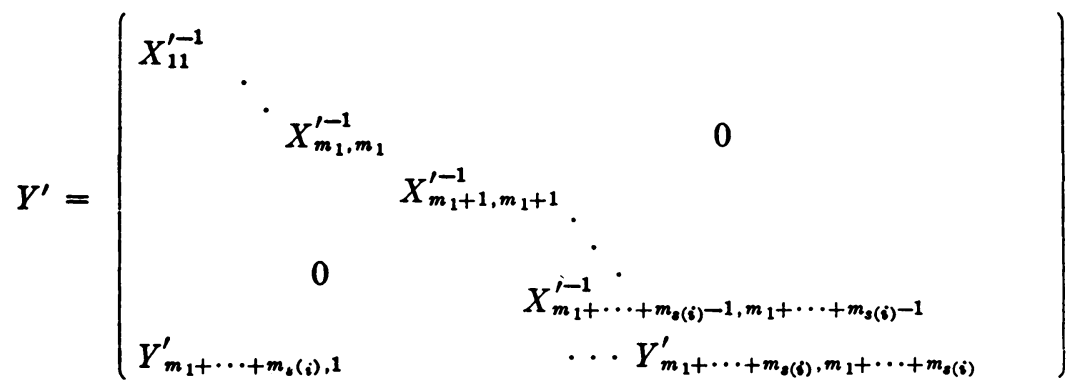

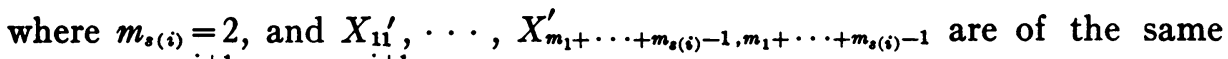
orders as $I_{1}^{i+1}, \cdots, I_{m_{1}+\cdots+m_{s(i)}-1}^{i+1}$ respectively. Theorems 10 and 11 now follow by induction.

9. Necessary and sufficient conditions for the equivalence of a set of 2 way matrices to a set of diagonal matrices. Two-way matrices $F_{1}, \cdots, F_{m}$ are equivalent under non-singular linear transformations to a set of diagonal

* This reduction is accomplished by rearranging the diagonal elements of $E_{i+1}$. Hence the reduction of $E_{i+1}$ can be accomplished in any field $\phi$. 
matrices if and only if $F_{1}, \cdots, F_{m}$ are equivalent to a set of canonical diagonal matrices $C_{1}, \cdots, C_{m}$ as given in Theorem 10 . We shall therefore prove Theorems 12 and 13 below.

Write

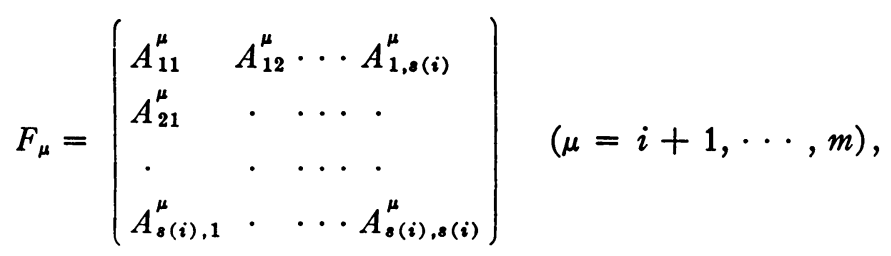

where $A_{11}^{\mu}, A_{22}^{\mu}, \cdots, A_{s(i), s(i)}^{\mu}$ are of the same orders as $I_{1}^{i}, I_{2}^{i}, \cdots, I_{s(i)}^{i}$ respectively. These last matrices are minors of the matrix $C_{i}$ of Theorem 10.

Theorem 12. Let $\Sigma=\left(C_{1}, \cdots, C_{i}, F_{i+1}, \cdots, F_{m}\right)$ be a set of 2-way matrices of order $n$ with elements in a field $\phi$. If $A_{b(i), 8(i)}^{\mu}=0$ for $\mu=i+1, \cdots, m$, the set $\Sigma$ is equivalent in $\phi$ to a set of diagonal matrices if and only if the following conditions are satisfied:

(a) $A_{\alpha \beta}^{\mu}=0 ; \alpha \neq \beta ; \alpha, \beta=1, \cdots, s(i)$, and $\mu=i+1, \cdots, m$.

(b) $A_{\gamma \gamma}^{i+1}, \cdots, A_{\gamma \gamma}^{m}$ are equivalent in $\phi$ for every $\gamma$ in the set $\gamma=1, \cdots$, $s(i)-1$ to a set of diagonal matrices under similar transformation.

Let $X, Y$ be given as in (10). We shall denote the matrices $F_{\mu}^{\prime}=X F_{\mu} Y$ by

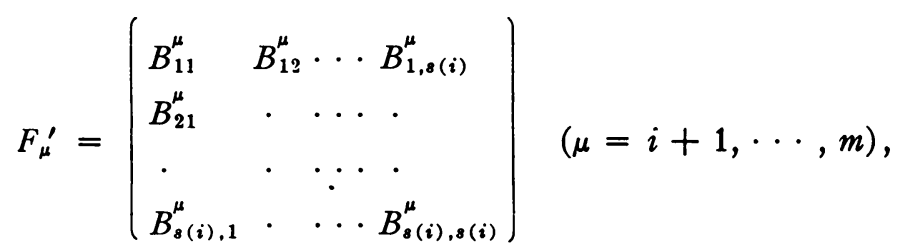

where the minors $B_{k l}^{\mu}$ are of the same orders as $A_{k l}^{\mu}$ for $k, l=1, \cdots, s(i)$. Now $B_{\alpha, s(i)}^{\mu}=X_{\alpha \alpha} A_{\alpha, s(i)}^{\mu} Y_{s(i), s(i)}, \alpha=1, \cdots, s(i)-1$. Also $B_{s(i), \alpha}^{\mu}=X_{s(i), s(i)} A_{s(i), \alpha}^{\mu} X_{\alpha \alpha}^{-1}$, $\alpha=1, \cdots, s(i)-1$. If the matrices $F_{\mu}^{\prime}$ are diagonal matrices it is necessary that $B_{\alpha, s(i)}^{\mu}=B_{s(i), \alpha}^{\mu}=0$ for $\alpha=1, \cdots, s(i)-1$, whence $A_{\alpha, s(i)}^{\mu}=A_{s(i), \alpha}^{\mu}=0$, $\alpha=1, \cdots, s(i)-1$. Substituting these results in the formulas for the remaining elements of $F_{\mu}$ we find we must also have $A_{\alpha \beta}^{\mu}=0$ for $\alpha \neq \beta ; \alpha, \beta=1, \cdots$, $s(i)-1$, and the set of matrices $A_{\gamma \gamma}^{i+1}, \cdots, A_{\gamma \gamma}^{m}$ must be equivalent for every $\gamma$, where $\gamma=1, \cdots, s(i)-1$, to a set of diagonal matrices under similar transformation. Necessary and sufficient conditions for such equivalence are given in Theorem 4. We have proved the necessity of the conditions of Theorem 12. They are also evidently sufficient.

If $A_{s(i), 8(i)}^{i+1}$ is of rank $r^{\prime} \neq 0$, the matrix $F_{i+1}$ can be reduced under transformations leaving $C_{1}, \cdots, C_{i}$ invariant to a new matrix $F_{i+1}$ in which 


$$
A_{s(i), s(i)}^{i+1}=\left[I_{s(i+1)-1}^{i+1}, 0\right],
$$

where $I_{s(i+1)-1}^{i+1}$ is a Kronecker delta of order $r^{\prime}$. Write

$$
A_{\rho, 8(i)}^{i+1}=\left(A_{\rho, 8(i)}^{[1]} A_{\rho, 8(i)}^{[2]}\right), \quad A_{s(i), \rho}^{i+1}=\left(\begin{array}{c}
A_{s(i), \rho}^{[1]} \\
A_{s(i), \rho}^{[2]}
\end{array}\right),
$$

where $\rho=1, \cdots, s(i)-1$, and the $A_{\rho, s(i)}^{[1]}$ have $r^{\prime}$ columns while the $A_{s(i), \rho}^{[1]}$ have $r^{\prime}$ rows. We can now state

THEOREM 13. Let the minor $A_{s(i),(i)}^{i+1}$ of $F_{i+1}$ be reduced as in (11). The set of matrices $\Sigma^{\prime}=\left(C_{1}, \cdots, C_{i}, F_{i+1}\right)$ is equivalent in a field $\phi$ to a set of diagonal matrices if and only if the following conditions are satisfied:

(a) $A_{a(i), \rho}^{[2]}=A_{\rho, 0(i)}^{[2]}=0$ for $\rho=1, \cdots, s(i)-1$.

(b) $A_{\alpha \beta}^{i+1}=A_{\alpha, \theta(i)}^{(1)} A_{s(i), \beta}^{[1]} \alpha \neq \beta ; \alpha, \beta=1, \cdots, s(i)-1$.

(c) The invariant factors of the matrices $A_{\alpha \alpha}^{i+1}-A_{\alpha, 8(i)}^{[1]} A_{s(i), \alpha}^{[1]}-\lambda I_{\alpha}, \alpha=1, \cdots$, $s(i)-1$, where the $I_{\alpha}$ are Kronecker deltas, factor into distinct linear factors in $\phi$.

The non-singular matrices $X_{s(i), 8(i)}, Y_{s(i), s(i)}$ which transform $A_{s(i),(i)}^{i+1}$ $=\left[I_{s(i+1)-1}^{i+1}, 0\right]$ into itself, so that $X_{s(i), s(i)} A_{s(i), s(i)}^{i+1} Y_{s(i), s(i)}=A_{s(i) s(i)}^{i+1}$, are of the form

$$
X_{s(i), s(i)}=\left(\begin{array}{cc}
W_{11} & W_{12} \\
0 & W_{22}
\end{array}\right), \quad Y_{s(i), s(i)}=\left(\begin{array}{cc}
W_{11}^{-1} & 0 \\
V_{21} & V_{22}
\end{array}\right),
$$

where $W_{11}$ is a minor of the same order as $I_{s(i+1)-1}^{i+1}$. If the set $\Sigma^{\prime}$ is to be equivalent to a set of diagonal matrices, it must be equivalent to a set $C_{1}, \cdots, C_{i+1}$, where $S_{s(i)}^{i}=A_{s(i),(i)}^{i+1}$. The matrix $S_{s(i)}^{i}$ is a minor of $C_{i+1}$ as in Theorem 10 . The matrix $F_{i+1}$ must then be equivalent to a matrix $C_{i+1}$ under transformations $X, Y$ which leave the set $\Sigma^{\prime \prime}=\left(C_{1}, \cdots, C_{i}, A_{s(i), s(i)}^{i+1}\right)$ invariant. If such matrices $X, \dot{Y}$ exist, then $X^{-1}, Y^{-1}$ also leaves $\Sigma^{\prime \prime}$ invariant. If $X F_{i+1} Y=C_{i+1}$, then $F_{i+1}=X^{-1} C_{i+1} Y^{-1}$. Hence the set $\Sigma^{\prime}$ is equivalent to a set $C_{1}, \cdots, C_{i+1}$ if and only if there exist matrices $X, Y$ leaving $\Sigma^{\prime \prime}$ invariant such that

$$
F_{i+1}=X C_{i+1} Y \text {. }
$$

If there are to be matrices $X, Y, C_{i+1}$ such that (12) is satisfied, it is readily seen by equating matrices that the following conditions must be satisfied:

$$
\begin{aligned}
& A_{s(i), 1}^{i+1}=\left(\begin{array}{cc}
W_{11} & 0 \\
0 & 0
\end{array}\right) Y_{s(i), 1} ; \cdots ; A_{s(i), s(i)-1}^{i+1}=\left(\begin{array}{cc}
W_{11} & 0 \\
0 & 0
\end{array}\right) Y_{s(i), s(i)-1} \\
& A_{1, s(i)}^{i+1}=X_{1, s(i)}\left(\begin{array}{cc}
W_{11}^{-1} & 0 \\
0 & 0
\end{array}\right) ; \cdots ; A_{s(i)-1, s(i)}^{i+1}=X_{s(i)-1,8(i)}\left(\begin{array}{cc}
W_{11}^{-1} & 0 \\
0 & 0
\end{array}\right) .
\end{aligned}
$$


Let

$$
Y_{s(i), \rho}=\left(\begin{array}{c}
Y_{\rho}^{\prime} \\
Y_{\rho}^{\prime \prime}
\end{array}\right), \quad X_{\rho, s(i)}=\left(X_{\rho}^{\prime} X_{\rho}^{\prime \prime}\right)
$$

for $\rho=1, \cdots, s(i)-1$, where $Y_{\rho}^{\prime}$ has $r^{\prime}$ rows and $X_{\rho}^{\prime}$ has $r^{\prime}$ columns. By (13) and (14) we have

$$
A_{8(i), \rho}^{[2]}=A_{\rho, 8(i)}^{[2]}=0 \quad(\rho=1, \cdots, s(i)-1) .
$$

Further

$$
\begin{aligned}
& Y_{1}^{\prime}=W_{11}^{-1} A_{8(i), 1}^{[1]}, \cdots, Y_{s(i)-1}^{\prime}=W_{11}^{-1} A_{s(i), 8(i)-1}^{[1]} ; \\
& X_{1}^{\prime}=A_{1, s(i)}^{[1]} W_{11}, \cdots, X_{s(i)-1}^{\prime}=A_{s(i)-1,8(i)}^{[1]} W_{11} .
\end{aligned}
$$

Substituting (16) in $X C_{i+1} Y$, and using (15), we get

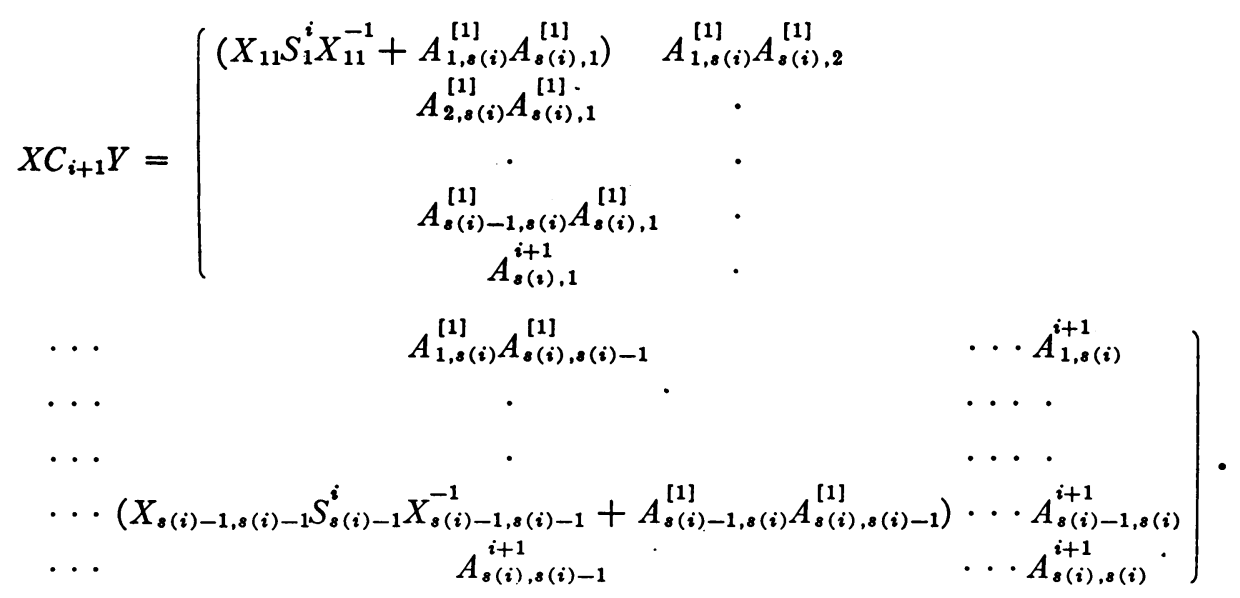

To have the above matrix equal to $F_{i+1}$ we must have

$$
A_{\alpha \beta}^{i+1}=A_{\alpha, s(i)}^{[1]} A_{s(i), \beta}^{[1]} ; \quad \alpha \neq \beta ; \quad \alpha, \beta=1, \cdots, s(i)-1 .
$$

Also $X_{\alpha \alpha} S_{\alpha}^{i} X_{\alpha \alpha}^{-1}+A_{\alpha, 8(i)}^{[1]} A_{8(i), \alpha}^{[1]}=A_{\alpha \alpha}^{i+1}$, whence $A_{\alpha \alpha}^{i+1}-A_{\alpha, 8(i)}^{[1]} A_{\delta(i), \alpha}^{[1]}$ must each be equivalent under similar transformation to a diagonal matrix for every $\alpha$ where $\alpha=1, \cdots, s(i)-1$. The necessity of condition (c) of the theorem now follows from the lemma, $\$ 4$.

We have proved the necessity of the conditions of Theorem 13 . The suffciency of these conditions is evident.

10. Necessary and sufficient conditions for the equivalence of a set of $p$-way matrices, $p \geqq 3$, to a set of diagonal matrices. We shall now state the analogues of Theorems 10 and 11 for $p \geqq 3$. 
Let $C_{1}, \cdots, C_{s}$ be $p$-way matrices, $p \geqq 3$, of orders $n_{1}, \cdots, n_{s}$ respectively. Let a $p$-way matrix $C$ of order $n_{1}+\cdots+n_{s}$ be constructed in a $p$-space by placing the matrices $C_{1}, \cdots, C_{s}$ in a non-overlapping fashion on the principal diagonal of $C$ so that the principal diagonals of $C_{1}, \cdots, C_{8}$ form the principal diagonal of $C$, and let the elements of $C$ not in these "minors" be zero. The matrix $C$ will be denoted, as in the 2-way case, by $C=\left[C_{1}, \cdots, C_{8}\right]$. Let the quantities $\lambda, \delta$ with or without subscripts and superscripts denote a $p$-way non-singular diagonal matrix and a $p$-way $\delta$-matrix respectively. We can now state

THEOREM 14. Let $S=\left[E_{1}, \cdots, E_{q}\right]$ be a set of p-way diagonal matrices, $p \geqq 3$, of order $n$ with elements in a field $\phi$. The set $S$ is equivalent in $\phi$ to a set $S^{\prime}=\left(C_{1}, \cdots, C_{q}\right)$, where

$$
C_{1}=\left[\delta_{1}, 0\right], \quad C_{2}=\left[S_{1}, T_{1}\right],
$$

and

$$
S_{1}=\left[\lambda_{1}{ }^{2}, 0\right], \quad T_{1}=\left[\delta_{2}, 0\right]
$$

the matrix $S_{1}$ is of the same order as $\delta_{1}$; in general any consecutive pair in the set $S^{\prime}$ is of the form

$$
\begin{aligned}
C_{\alpha} & =\left[\lambda_{1}^{\alpha}, 0, \lambda_{2}^{\alpha}, 0, \cdots, \lambda_{\sigma(\alpha)}^{\alpha}, 0, \delta_{\alpha}, 0\right], \\
C_{\alpha+1} & =\left[Q_{1}^{\alpha}, R_{1}^{\alpha}, Q_{2}^{\alpha}, R_{2}^{\alpha}, \cdots, Q_{\sigma(\alpha)}^{\alpha}, R_{\sigma(\alpha)}^{\alpha}, S_{\alpha}, T_{\alpha}\right],
\end{aligned}
$$

where

$$
\begin{array}{rlrl}
Q_{q}^{\alpha} & =\left[\lambda_{2 q-1}^{\alpha+1}, 0\right] & & (q=1, \cdots, \sigma(\alpha)), \\
R_{r}^{\alpha} & =\left[\lambda_{2 r}^{\alpha+1}, 0\right] & & (r=1, \cdots, \sigma(\alpha)), \\
S_{\alpha} & =\left[\lambda_{\sigma(\alpha+1)}^{\alpha+1}, 0\right], & \\
T_{\alpha} & =\left[\delta_{\alpha+1}, 0\right], &
\end{array}
$$

and $\sigma(\alpha)=2^{\alpha-1}-1, \alpha \geqq 2$, and the minors $Q_{1}^{\alpha}, R_{1}^{\alpha}, \cdots, Q_{\sigma(\alpha)}^{\alpha}, R_{\sigma(\alpha)}^{\alpha}, S_{\alpha}, T_{\alpha}$ are of the same orders as the corresponding minors $\lambda_{1}^{\alpha}, 0, \cdots, \lambda_{\sigma(\alpha)}^{\alpha}, 0, \delta_{\alpha}, 0$ of $C_{\alpha}$.

Let $i, j, \cdots, k, l$ be the indices of the matrices $C_{1}, \cdots, C_{q}$. As we prove Theorem 14 we shall also prove

Theorem 15. Let $A^{q}=\left(a_{i \beta}^{q}\right), B^{q}=\left(b_{j \gamma}^{q}\right), \cdots, C^{q}=\left(c_{k_{\tau}}^{q}\right), D^{q}=\left(d_{l \varepsilon}^{q}\right)$ denote matrices which, under non-singular linear transformations with these matrices on the indices $i, j, \cdots, k, l$ of the matrices in the set $S^{\prime}=\left(C_{1}, \cdots, C_{q}\right)$, leave the set $S^{\prime}$ invariant or at most reorder the elements of the minors $\lambda_{m}^{q}, m=1, \cdots, \sigma(q)$, of $C_{q}$ independently for each $m$. The matrices $A^{q}, \cdots, D^{q}$ are of the form 


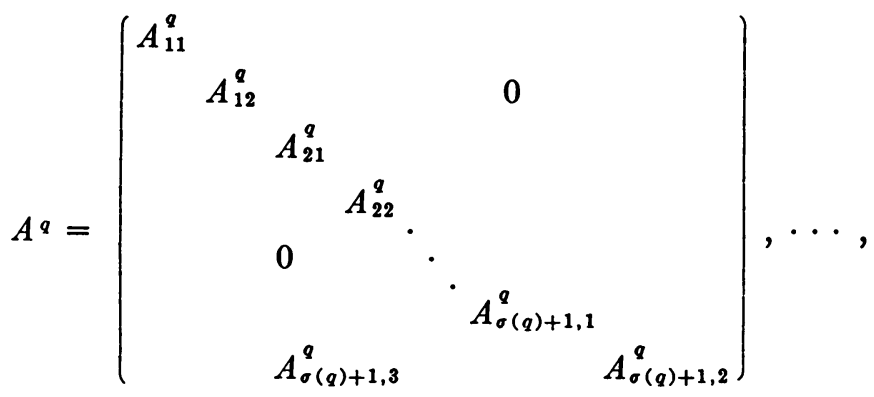

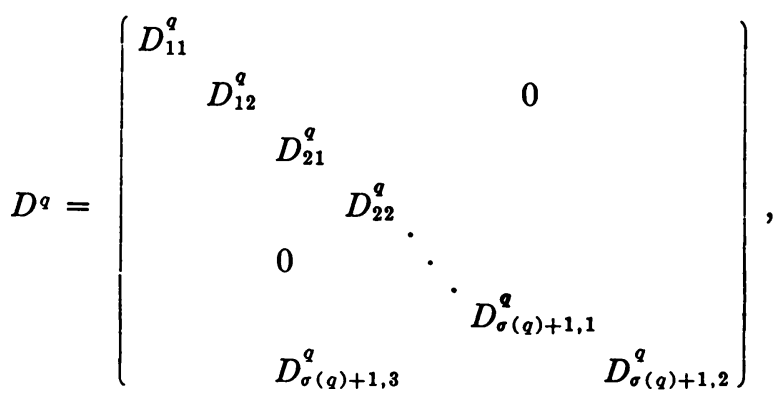

where $D_{\gamma 1}^{q}=A_{\gamma 1}^{q^{-1}} B_{\gamma 1}^{q^{-1}} \cdots C_{\gamma 1}^{q^{-1}}$ for $\gamma=1, \cdots, \sigma(q)+1 ; D_{\gamma 2}^{q}=A_{\gamma 2}^{q^{-1}} B_{\gamma 2}^{q^{-1}} \cdots C_{\gamma 2}^{q^{-1}}$ for $\gamma=1, \cdots, \sigma(q)$; and corresponding minors in the sets $\left(A_{11}^{q}, \cdots, A_{\sigma(q)+1,1}^{q}\right)$, $\cdots,\left(D_{11}^{q}, \cdots, D_{\sigma(q)+1,1}^{q}\right)$ are of the same orders as $\lambda_{1}^{q}, \lambda_{2}^{q}, \cdots, \lambda_{\sigma(q)}^{q}, \delta_{q} r e-$ spectively; the remaining minors of $A^{q}, \cdots, D^{a}$ are of the same orders as the corresponding zero minors of $C_{q}$; also, every minor except the last on the diagonal of each matrix $A^{q}, \cdots, D^{q}$ as written in (17) is a diagonal matrix. The matrices $A^{q}, \cdots, D^{a}$ may also be of the types obtained from those given in (17) by simultaneous interchanges of the rows and simultaneous interchanges of the columns of the minors in each of the sets $\left(A_{\sigma(q)+1,1}^{q}, \cdots, D_{\sigma(q)+1,1}^{q}\right),\left(A_{\alpha \beta}^{q}, \cdots, D_{\alpha \beta}^{q}\right)$; $\alpha=1, \cdots, \sigma(q) ; \beta=1,2$, these interchanges being made independently for each set.

If there are $u_{1}^{1}$ non-vanishing elements on the diagonal of $E_{1}$, it is evident at once that $E_{1}$ is equivalent to $C_{1}$, where $\delta_{1}$ is of order $u_{1}^{1}$.

Denote $C_{\alpha}$ by $\left(c_{i j}^{\alpha} \cdots k l\right)$ for every $\alpha$. If $\left(c_{i j}^{1} \cdots k l a_{i \beta}^{1} b_{j \gamma}^{1} \cdots c_{k \tau}^{1} d_{l e}^{1}\right)=\left(c_{i j}^{1} \cdots k l\right)$, then

$$
\left(\sum_{\alpha=1}^{u_{1}^{1}} a_{\alpha \beta}^{1} b_{\alpha \gamma}^{1} \cdots c_{\alpha \tau}^{1} d_{\alpha \epsilon}^{1}\right)=0
$$

for $\gamma, \cdots, \tau, \epsilon=1, \cdots, n$ and $\beta=u_{1}^{1}+1, \cdots, n$. By (18), $Q P=0$ where 


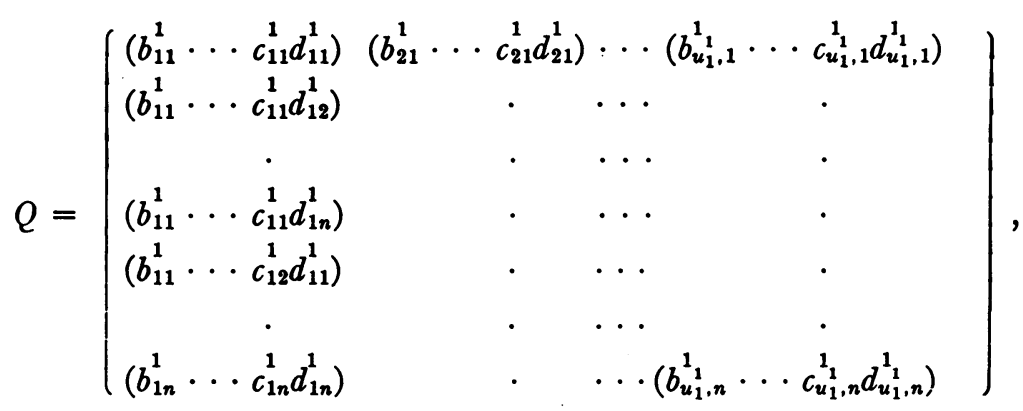

$$
\begin{aligned}
& P=\left(\begin{array}{cccc}
a_{1, u_{1}+1}^{1} & a_{1, u_{1}+2}^{1} & \cdots & a_{1, n}^{1} \\
a_{2, u_{1}+1}^{1} & \cdot & \cdots & . \\
\cdot & \cdot & \cdots & . \\
a_{u_{1}, u_{1}+1}^{1} & \cdot & \cdots & a_{u_{1}, n}^{1}
\end{array}\right) .
\end{aligned}
$$

$Q$ is a minor of the display $G=\left(g_{\kappa \lambda}\right) ; \kappa=\gamma, \cdots, \tau, \epsilon ; \lambda=j, \cdots, l$ of the matrix $J=B^{1} \times \cdots \times C^{1} \times D^{1}$. By a lemma proved in another paper* by the author, $G$ is non-singular since $B^{1}, C^{1}, \cdots, D^{1}$ are non-singular. Hence $Q$ is nonsingular on its columns, and $P=0$. Similarly,

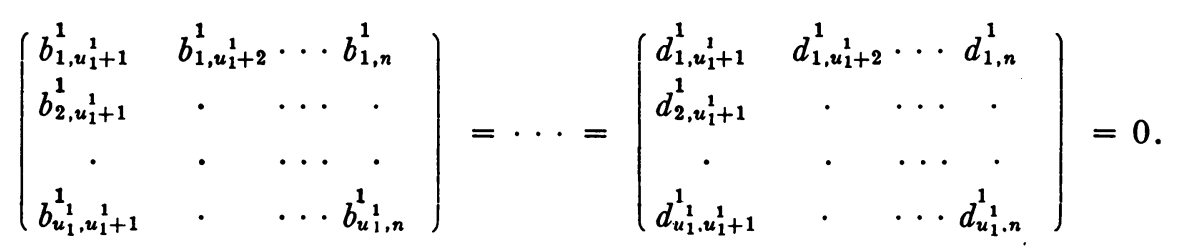

Also

$$
\left(\sum_{\alpha=1}^{u_{1}^{2}} a_{\alpha \beta}^{1} b_{\alpha \gamma}^{1} \cdots d_{\alpha \epsilon}^{1}\right)=\delta,
$$

where $\delta$ is a $\delta$-matrix on $(\beta, \gamma, \cdots, \tau, \epsilon)$ and $\beta, \gamma, \cdots, \epsilon=1, \cdots, u_{1}^{1}$. Applying Theorem 1 we obtain

$$
A^{1}=\left(\begin{array}{cccc}
a_{11}^{1} & & & \\
& \cdot & 0 \\
0 & \cdot a_{u_{1}, u_{1}^{1}}^{1} & \\
A^{\prime} & & A^{\prime \prime}
\end{array}\right), \quad B^{1}=\left(\begin{array}{ccc}
b_{11}^{2} & & \\
& 0 \\
0 & b_{u_{1}, u_{1}^{1}}^{1} \\
B^{\prime} & & B^{\prime \prime}
\end{array}\right), \cdots
$$

* Composition and rank of n-way matrices and multilinear forms, Annals of Mathematics, vol. 35 (1934), p. 625. 


$$
D^{1}=\left(\begin{array}{cccc}
d_{11}^{1} & & & \\
& \ddots & 0 & \\
0 & d_{u_{1}, u_{1}^{1}}^{1} & \\
D^{\prime} & & D^{\prime \prime}
\end{array}\right),
$$

or matrices obtained from $A^{1}, B^{1}, \cdots, D^{1}$ above by simultaneous interchanges of the first $u_{1}^{1}$ rows and $u_{1}^{1}$ columns of $A^{1}, \cdots, D^{1}$.

Assume that $E_{1}, \cdots, E_{\alpha-1}$ have been reduced to $C_{1}, \cdots, C_{\alpha-1}$, and the matrices

$$
A^{\alpha-1}=\left(a_{i \beta}^{\alpha-1}\right), B^{\alpha-1}=\left(b_{j \gamma}^{\alpha-1}\right), \cdots, D^{\alpha-1}=\left(d_{l e}^{\alpha-1}\right),
$$

which satisfy the relations

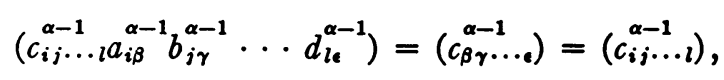

are of the form

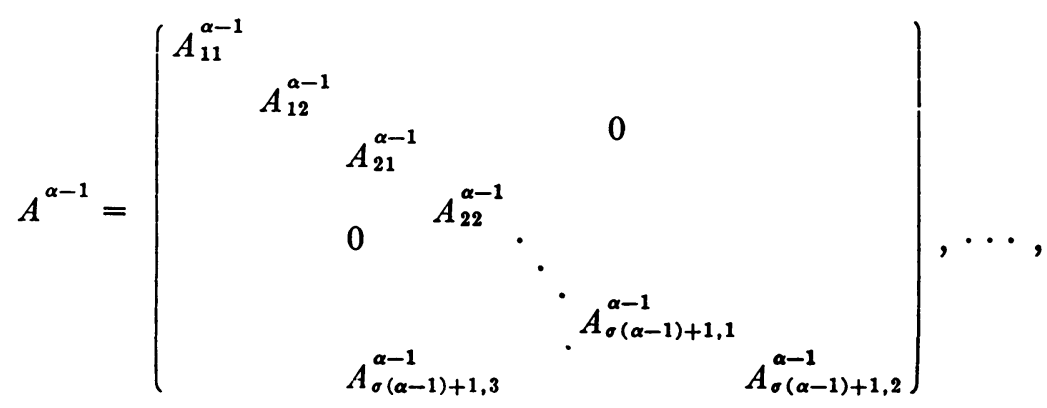

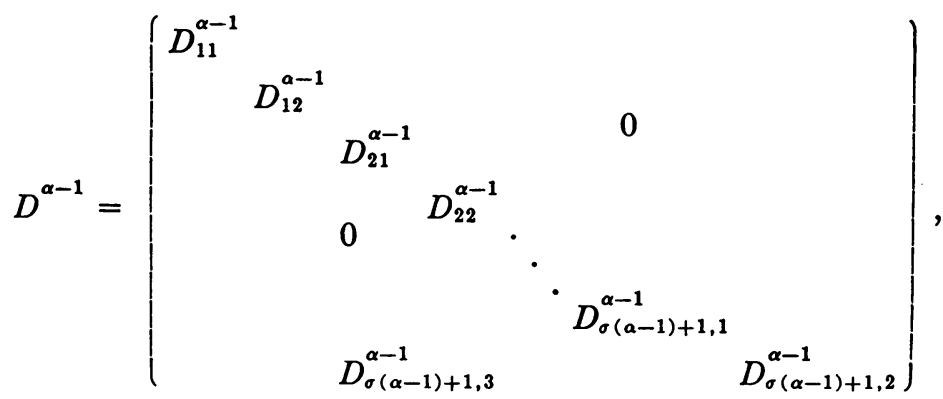

where these matrices satisfy the properties mentioned for $A^{q}$ in Theorem 15 . It is evident at once that $E_{\alpha}$ can be reduced under transformations with $A^{\alpha-1}, \cdots, D^{\alpha-1}$ to a matrix of type $C_{\alpha}$.

We shall now restrict the matrices $A^{\alpha-1}, \cdots, D^{\alpha-1}$ so that

$$
\left(c_{i j \ldots l}^{\alpha} a_{i \beta}^{\alpha-1} b_{j \gamma}^{\alpha-1} \cdots d_{l \epsilon}^{\alpha-1}\right)=C_{\alpha} .
$$


Let the orders of the minors $\lambda_{1}^{\alpha}, \lambda_{2}^{\alpha}, \cdots, \lambda_{\sigma(\alpha)}^{\alpha}, \delta_{\alpha}$ be denoted by $u_{1}^{\alpha}, u_{2}^{\alpha}, \cdots$, $u_{\left.\sigma_{(\alpha)}\right)}^{\alpha}, u_{\sigma(\alpha)+1}^{\alpha}$ respectively; and the orders of the zero minors between these minors by $v_{1}^{\alpha}, \cdots, v_{\sigma(\alpha)+1}^{\alpha}$ respectively. Let $\Sigma_{\alpha}=u_{1}^{\alpha}+\cdots+u_{\sigma(\alpha)}^{\alpha}+v_{1}^{\alpha}$ $+\cdots+v_{\sigma(\alpha)}^{\alpha}$. By (19),

$$
\left(\sum_{x=\Sigma_{\alpha}+1}^{\Sigma_{\alpha}+u \sigma^{\alpha}(\alpha)+1} a_{x \beta}^{\alpha-1} b_{x \gamma}^{\alpha-1} \cdots d_{x \epsilon}^{\alpha-1}\right)=0
$$

if $\gamma, \cdots, \epsilon=\Sigma_{\alpha}+1, \cdots, n$, and $\beta=1, \cdots, \Sigma_{\alpha}$. By (20) $\Gamma_{1} \Gamma_{2}=0$, where

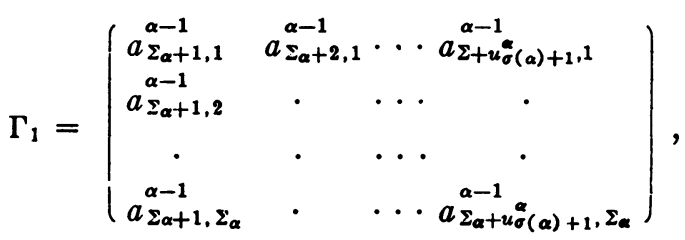

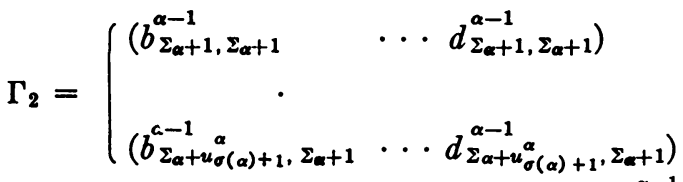

$$
\begin{aligned}
& \cdots\left(b_{\Sigma_{\alpha+1, n}}^{\alpha-1} \quad \cdots d_{\Sigma_{\alpha+1, n}^{\alpha-1}}^{\alpha-1}\right) \\
& \text {... }
\end{aligned}
$$

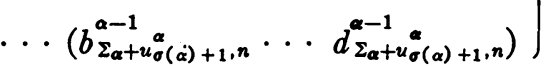

Since $B^{\alpha-1}, \cdots, D^{\alpha-1}$ are to be taken non-singular, the minors

$$
\begin{aligned}
& B_{\sigma(\alpha-1)+1,2}^{\alpha-1}=\left(\begin{array}{cccc}
b_{\Sigma_{\alpha+1}-\Sigma_{\alpha+1}}^{\alpha-1} & b_{\Sigma_{\alpha+1, \Sigma_{\alpha+2}}^{\alpha-1}} \cdots & b_{\Sigma_{\alpha+1, n}}^{\alpha-1} \\
b_{\Sigma_{\alpha+2, \Sigma_{\alpha+1}}-1}^{\alpha+1} & \cdot & \cdots & \cdot \\
\cdot & \cdot & \cdots & \cdot \\
b_{n, \Sigma_{\alpha+1}}^{\alpha-1} & \cdot & \cdots & b_{n, n}^{\alpha-1}
\end{array}\right), \cdots,
\end{aligned}
$$

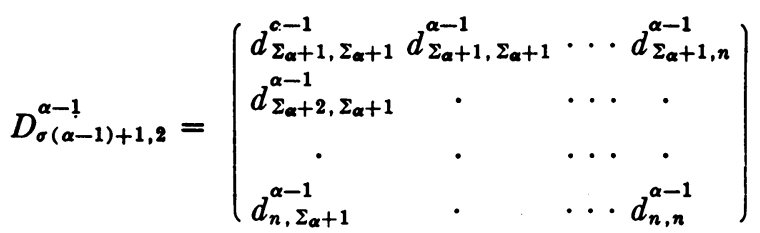

are non-singular. By the lemma of another paper mentioned above, ${ }^{*}$ the display $\Omega=\left(\omega_{\lambda_{\mu}}\right)$ is non-singular, where $\Omega=B_{\sigma(\alpha-1)+1,2}^{\alpha-1} \times \cdots \times D_{\sigma(\alpha-1)+1,2}^{\alpha-1}$ and $\lambda=j \cdots k l ; \mu=\gamma \cdots \tau \epsilon ; \gamma, \cdots, \tau, \epsilon, j, \cdots, k, l=\Sigma_{\alpha}+1, \cdots, n$. The

* Composition and rank of n-way matrices and multilinear forms, Annals of Mathematics, vol. 35 (1934), p. 625. 
matrix $\Gamma_{2}$ is a minor of $\Omega$ consisting of certain rows of $\Omega$ and is therefore nonsingular on its rows. It follows that $\Gamma_{1}=0$. Similarly

$$
\left(\begin{array}{ccc}
b_{\Sigma_{\alpha+1,1}^{\alpha-1}}^{\alpha-1} & \cdots & b_{\Sigma_{\alpha+u_{\sigma(\alpha)+1}}^{\alpha-1}}^{\alpha} \\
\cdot & \cdots & \cdot \\
b_{\Sigma_{\alpha+1, \Sigma_{\alpha}}^{\alpha-1}} & \cdots & b_{\Sigma_{\alpha+u_{\sigma(\alpha)+1}, \Sigma_{\alpha}}^{\alpha}}^{\alpha}
\end{array}\right), \cdots,\left(\begin{array}{ccc}
d_{\Sigma_{\alpha+1,1}}^{\alpha-1} & \cdots & d_{\Sigma_{\alpha+u_{\sigma}(\alpha)+1,1}^{\alpha-1}}^{\alpha} \\
\cdot & \cdots & \cdot \\
d_{\Sigma_{\alpha+1, \Sigma_{\alpha}}^{\alpha-1}}^{\alpha} & \cdots & d_{\Sigma_{\alpha+u_{\sigma}(\alpha)+1}^{\alpha}, \Sigma_{\alpha}}^{\alpha}
\end{array}\right)
$$

are zero

It follows from (19) that

$$
\left(\sum_{\lambda=\Sigma_{\alpha+1}}^{\Sigma_{\alpha+1}+u_{\sigma}(\alpha)+1} a_{\lambda \beta}^{\alpha-1} \cdots d_{\lambda \epsilon}^{\alpha-1}\right)=0
$$

if $\beta=\Sigma_{\alpha}+u_{\sigma(\alpha)+1}^{\alpha}+1, \Sigma_{\alpha}+u_{\sigma(\alpha)+1}^{\alpha}+2, \cdots, n ; \gamma, \cdots, \tau, \epsilon=\Sigma_{\alpha}+1, \cdots, n$.

By (21), $\Gamma_{3} \Gamma_{2}=0$, where

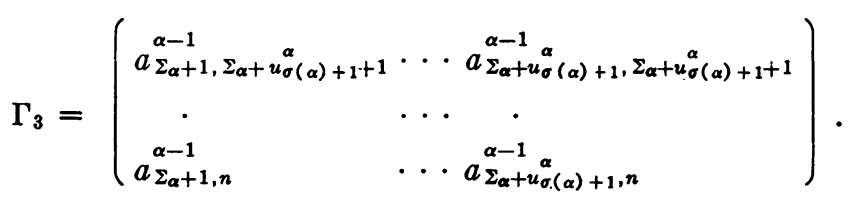

Again, since $\Gamma_{2}$ is non-singular on its rows, $\Gamma_{3}=0$. Similarly,

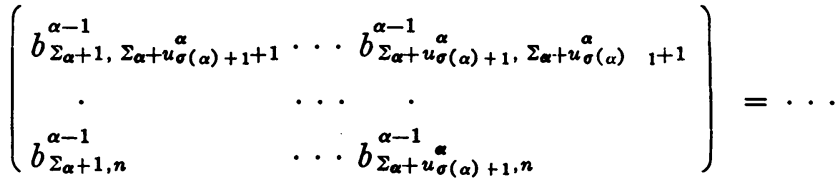

$$
\begin{aligned}
& =\left(\begin{array}{ccc}
d_{\Sigma_{\alpha+1}}^{\alpha-1} \Sigma_{\alpha+u_{\sigma(\alpha)+1+1}}^{\alpha} & \cdots & d_{\Sigma_{\alpha+u_{\sigma(\alpha)+1}}^{\alpha-1} \Sigma_{\alpha+u_{\sigma(\alpha)+1+1}}^{\alpha}}^{\alpha-1} \\
\cdot & \cdots & \cdot \\
d_{\Sigma_{\alpha+1, n}^{\alpha-1}}^{\alpha-1} & \cdots & d_{\Sigma_{\alpha+u_{\sigma(\alpha)+1, n}}^{\alpha-1}}^{\alpha}
\end{array}\right)=0 .
\end{aligned}
$$

Further if $\beta, \gamma, \cdots, \epsilon=\Sigma_{\alpha}+1, \cdots, \Sigma_{\alpha}+u_{\sigma(\alpha)+1}^{\alpha}$,

$$
\left(\sum_{\lambda=\Sigma_{\alpha}+1}^{\Sigma_{\alpha}+u_{\sigma}(\alpha)+1+1} a_{\lambda \beta}^{\alpha-1} \cdots d_{\lambda \epsilon}^{\alpha-1}\right)=\delta,
$$

where $\delta$ is a $\delta$-matrix on $(\beta, \gamma, \cdots, \epsilon)$. Equations of type (22) have already been treated.*

In view of the above considerations, the matrices $A^{\alpha-1}, \cdots, D^{\alpha-1}$ which satisfy (19) are of the form $A^{\alpha}, \cdots, D^{\alpha}$ as obtained from (17) by writing $q=\alpha$, and satisfy the properties of Theorem 15 for these matrices.

Theorems 14 and 15 follow by induction.

* See Theorem 1. 
We shall now prove an analogue of Theorems 12 and 13. Let $C_{\alpha 1}=\left(c_{\beta \gamma}^{\prime} \ldots \epsilon\right)$ be a matrix obtained from $C_{\alpha}$ of Theorem 14 by replacing $\delta_{\alpha}$ by a zero matrix. We have

TheOREm 16. Let $\Sigma=\left(C_{1}, \cdots, C_{\alpha-1}, K_{\alpha}\right)$ be a set of $p$-way, $p \geqq 3$, matrices of order $n$ with elements in a field $\phi$, where $C_{1}, \cdots, C_{\alpha-1}$ are canonical diagonal matrices as given in Theorem 14. If the set $\Sigma$ is equivalent in $\phi$ to a set of diagonal matrices, the orders of $\lambda_{1}{ }^{\alpha}, \lambda_{2}{ }^{\alpha}, \cdots, \lambda_{\sigma(\alpha)}^{\alpha}, \delta_{\alpha}$ and values of the non-vanishing elements of $C_{\alpha 1}$ can be chosen so that $K_{\alpha}-C_{\alpha 1}$ is a non-singular matrix of order $r$, where $r$ is the order of $\delta_{\alpha}$, or such a matrix bordered by zeros; and conversely.

Let $K_{\alpha}=\left(k_{\beta \gamma} \ldots \epsilon\right)$. If $\Sigma$ is equivalent to a set of diagonal matrices, there exist matrices $A^{\alpha-1}=\left(a_{i \beta}^{\alpha-1}\right), \cdots, D^{\alpha-1}=\left(d_{l e}^{\alpha-1}\right)$, leaving $C_{1}, \cdots, C_{\alpha-1}$ invariant, and minors $\lambda_{1}{ }^{\alpha}, \cdots, \lambda_{\sigma(\alpha)}^{\alpha}, \delta_{\alpha}$ such that

$$
\left(k_{\beta \gamma} \ldots \epsilon\right)=\left(c_{i j \ldots l}^{\alpha} a_{i \beta}^{\alpha-1} b_{j \gamma}^{\alpha-1} \cdots d_{l \epsilon}^{\alpha-1}\right),
$$

whence

$$
\begin{aligned}
& \left(k_{\beta} \ldots \epsilon\right)=\left(\sum_{\lambda=1}^{u_{1}^{\alpha}} c_{\lambda \ldots \lambda}^{\alpha} a_{\lambda \beta}^{\alpha-1} \cdots d_{\lambda \epsilon}^{\alpha-1}+\sum_{\lambda=u_{1}^{\alpha}+v_{1}+1}^{u_{1}^{\alpha}+\sum_{1}^{\alpha}+u_{2}^{\alpha}} c_{\lambda \ldots \lambda}^{\alpha} \underset{\lambda \beta}{\alpha-1} \cdots d_{\lambda \epsilon}^{\alpha-1}\right.
\end{aligned}
$$

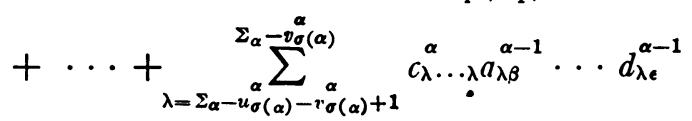

$$
\begin{aligned}
& \left.+\sum_{\lambda=\Sigma_{\alpha+1}}^{\Sigma_{\alpha+1}+u_{\sigma}^{\alpha}(\alpha)+1} a_{\lambda \beta}^{\alpha-1} \cdots d_{\lambda \epsilon}^{\alpha-1}\right)
\end{aligned}
$$

where the $u$ 's and $v$ 's are as defined in the proof of Theorems 14 and 15. At once

$$
K_{\alpha}-C_{\alpha 1}=\left(\sum_{\lambda=\Sigma_{\alpha+1}}^{\Sigma_{\alpha+u_{\alpha}}^{\alpha}(\alpha)+1} a_{\lambda \beta}^{\alpha-1} \cdots d_{\lambda \epsilon}^{\alpha-1}\right) .
$$

If we wish to test the equivalence of a set $C_{1}, \cdots, C_{\alpha-1}, K_{\alpha}, \cdots, K_{m}$ to diagonal matrices, we determine whether or not $C_{1}, \cdots, C_{\alpha-1}, K_{\alpha}$ is equivalent to a set $C_{1}, \cdots, C_{\alpha}$. If so, let $K_{\alpha+1}, \cdots, K_{m}$ go into $K_{\alpha+1}^{\prime}, \cdots, K_{m}^{\prime}$ under transformation of the set $C_{1}, \cdots, C_{\alpha-1}, K_{\alpha}$ to $C_{1}, \cdots, C_{\alpha}$. The matrices $C_{1}, \cdots, C_{\alpha-1}, K_{\alpha}, \cdots, K_{m}$ are equivalent under non-singular linear transformations to diagonal matrices if and only if $C_{1}, \cdots, C_{\alpha}, K_{\alpha+1}^{\prime}, \cdots, K_{m}^{\prime}$ are equivalent to diagonal matrices. We reapply Theorem 16 to $C_{1}, \cdots, C_{\alpha}$, $K_{\alpha+1}^{\prime}$. This process can be continued until we arrive at a canonical set $C_{1}, \cdots, C_{m}$.

In Theorem 14 we obtained canonical forms of $p$-way diagonal matrices, $p \geqq 3$, and in Theorem 16 necessary and sufficient conditions for the equiva- 
lence of $C_{1}, \cdots, C_{\alpha-1}, K_{\alpha}$ to canonical diagonal matrices. Since a set of matrices is equivalent to a set of diagonal matrices if and only if it is equivalent to a set of canonical diagonal matrices, we have derived necessary and suffcient conditions for the equivalence of a set of $p$-way matrices, $p \geqq 3$, to a set of diagonal matrices.

Theorem 16 is in general difficult to apply. However, it is given here since no better equivalent theorem has been found.

\section{Chapter III. Factorization of $p$-way matrices into 3-WAY MATRICES}

11. Introduction. Necessary and sufficient conditions for a matrix $A=\left(a_{k_{1}} \cdots k_{p}\right)$ to be of the form $\left(a_{\alpha k_{1}}^{(1)} \cdots a_{\alpha k_{p}}^{(p)}\right)$, where $\left(a_{\alpha k_{1}}^{(1)}\right), \cdots,\left(a_{\alpha k_{p}}^{(p)}\right)$ are matrices non-singular on the index $\alpha$, are given in chapter I. In the present chapter necessary and sufficient conditions are obtained for a matrix $A=\left(a_{i j k_{1}} \cdots k_{p}\right)$ to be of the form $\left(a_{i j k_{1}}^{(1)} \cdots a_{i j k_{p}}^{(p)}\right), i$ not summed, $j$ summed, where the 3 -way matrices $\left(a_{i j k_{1}}^{(1)}\right), \cdots,\left(a_{i j k_{p}}^{(p)}\right)$ are non-singular on $i j$. The method of treatment covers the case where the index $j$ in the matrices above does not occur* and the matrices $\left(a_{i k_{1}}^{(1)}\right), \cdots,\left(a_{i k_{p}}^{(p)}\right)$ are non-singular on $i$.

In this chapter, as in the others, indices may be partitions consisting of more than one index.

12. Factorization into multiple composites. Let $\delta_{1}, \cdots, \delta_{m-1}$ designate the 2-way displays $\left(\delta_{T T^{\prime}}^{1}\right)=\left(\delta_{1 i_{1} i_{2}} \delta_{j_{1} j_{2}}\right), \quad\left(\delta_{T T^{\prime}}^{2}\right)=\left(\delta_{2 i_{1} i_{2}} \delta_{j_{1} j_{2}}\right), \cdots, \quad\left(\delta_{T T^{\prime}}^{m-1}\right)$ $=\left(\delta_{m-1, i_{1} i_{2}} \delta_{j_{1} j_{2}}\right)$ respectively, where $T=i_{1} j_{1}, T^{\prime}=i_{2} j_{2} ;\left(\delta_{j_{1} j_{2}}\right)$ is a Kronecker delta of order $t$, and $\left(\delta_{1 i_{1} i_{2}}\right), \cdots,\left(\delta_{m-1, i_{1} i_{2}}\right)$ are the $i$-layers of a $\delta$-matrix on $\left(i, i_{1}, i_{2}\right)$ of order $m$ obtained by setting $i=1, \cdots, m-1$ respectively. Let $\delta_{2}^{\prime}=\left(\delta_{1 i_{1} i_{2}}^{\prime} \delta_{j_{1} j_{2}}\right), \delta_{3}^{\prime}=\left(\delta_{2 i_{1} i_{2}}^{\prime} \delta_{j_{1} j_{2}}\right), \cdots, \delta_{m-1}^{\prime}=\left(\delta_{m-2, i_{1} i_{2}}^{\prime} \delta_{j_{1} j_{2}}\right)$, where $\left(\delta_{1 i_{1} i_{2}}^{\prime}\right)$, $\left(\delta_{2 i_{1} i_{2}}^{\prime}\right), \cdots,\left(\delta_{m-2, i_{1} i_{2}}^{\prime}\right)$ are the $i$-layers of the $\delta$-matrix $\left(\delta_{i i_{1} i_{2}}^{\prime}\right)$ on $\left(i, i_{1}, i_{2}\right)$ of order $(m-1)$ on each index obtained by setting $i=1, \cdots, m-2$ respectively.

Let $B_{1}, \cdots, B_{m-1}$ be a set of 2-way matrices of order $n=m t$ with elements in a field $\phi$. If the matrices $B_{1}, \cdots, B_{m-1}$ are equivalent under similar transformation in $\phi$ to $\delta_{1}, \cdots, \delta_{m-1}$, it is evidently necessary that the matrices $B_{i}$ be each equivalent under similar transformation in $\phi$ to $\delta_{i}$ for $i=1, \cdots, m-1$. Now $B_{1}$ is equivalent under similar transformation to $\delta_{1}$ if and only if $\left(B_{1}-\lambda I\right)$ has the same invariant factors $\dagger$ as $\left(\delta_{1}-\lambda I\right)$, where $I$ is a Kronecker delta. Assume that $B_{1}$ is equivalent to $\delta_{1}$ as demanded. By reduction of $B_{1}$ to $\delta_{1}$

* The matrix $A$ in this case is a generalization of the Scott product of two matrices. See M. Lecat, Abrégé de la Théorie des Déterminants à $n$ Dimensions, 1911, Introduction, p. xl.

† Dickson, Modern Algebraic Theories, p. 104. 
under similar transformation, the matrices $B_{2}, \cdots, B_{m-1}$ are transformed into a set $B_{2}^{\prime}, \cdots, B_{m-1}^{\prime}$. Let

$$
B_{s}^{\prime}=\left(\begin{array}{ll}
B_{s 11}^{\prime} & B_{s 12}^{\prime} \\
B_{s 21}^{\prime} & B_{s 22}^{\prime}
\end{array}\right) \quad(s=2,3, \cdots, m-1),
$$

where $B_{s 11}$ is a minor of order $t$.

THEOREM 17. The matrices $B_{1}, \cdots, B_{m-1}$ of order $n$ with elements in a field $\phi$ are equivalent under similar transformation in $\phi$ to a set $\delta_{1}, \cdots, \delta_{m-1}$ if and only if

$$
B_{s 11}^{\prime}=B_{s 21}^{\prime}=B_{s 12}^{\prime}=0 \quad(s=2, \cdots, m-1),
$$

and the set $B_{222}^{\prime}, B_{322}^{\prime}, \cdots, B_{m-1,22}^{\prime}$ is equivalent under similar transformation in $\phi$ to the set of $(n-t)$-order matrices $\delta_{2}^{\prime}, \delta_{3}^{\prime}, \cdots, \delta_{m-1}^{\prime}$.

The matrix $W$ which satisfies the relation $W \delta_{1} W^{-1}=\delta_{1}$ is of the form*

$$
W=\left(\begin{array}{cc}
W_{11} & 0 \\
0 & W_{22}
\end{array}\right)
$$

where $W_{11}$ is a square minor of $W$ of order $t$. Now for $s=2, \cdots, m-1$,

$$
W B_{s}^{\prime} W^{-1}=\left(\begin{array}{ll}
W_{11} B_{s 11}^{\prime} W_{11}^{-1} & W_{11} B_{s 12}^{\prime} W_{22}^{-1} \\
W_{22} B_{s 21}^{\prime} W_{11}^{-1} & W_{22} B_{s 22}^{\prime} W_{22}^{-1}
\end{array}\right) .
$$

Equating $W B_{s}^{\prime} W^{-1}$ above to $\delta_{s}$ we obtain the conditions of Theorem 17. The matrices $\delta_{2}^{\prime}, \delta_{3}^{\prime}, \cdots, \delta_{m-1}^{\prime}$ form an array like $\delta_{1}, \delta_{2}, \cdots, \delta_{m-1}$, whence the above process may be reapplied to $B_{222}^{\prime}, B_{322}^{\prime}, \cdots, B_{m-1,22}^{\prime}$, and $\delta_{2}^{\prime}, \delta_{3}^{\prime}, \cdots, \delta_{m_{-1}^{\prime}}^{\prime}$. Since $m$ is finite this process is a terminating one.

Let $\xi_{i}, i=1, \cdots, n-1$, now represent a diagonal matrix with the $i$ th element on the diagonal as the only non-vanishing element. For square 2-way matrices $B_{1}, \cdots, B_{n-1}$ of order $n$ to be equivalent under similar transformation to the set $\xi_{1}, \cdots, \xi_{n-1}$ it is in particular necessary that $\left(B_{1}-\lambda I\right)$ have the invariant factors $\lambda(\lambda-1), \lambda, \cdots, \lambda$. Assuming that this condition is satisfied, let the set $B_{1}, \cdots, B_{n-1}$ be reduced under similar transformation in $\phi$ to a set $\xi_{1}, B_{2}^{\prime}, \cdots, B_{n-1}^{\prime}$, where we write

$$
B_{i}^{\prime}=\left(\begin{array}{ll}
b_{i 11}^{\prime} & B_{i 12}^{\prime} \\
B_{i 21}^{\prime} & B_{i 22}^{\prime}
\end{array}\right) \quad(i=2, \cdots, n-1),
$$

where $b_{i 11}^{\prime}$ is a single element. Letting $n=m t, t=1$ in Theorem 17 , we have the

* Turnbull and Aitken, Introduction to the Theory of Canonical Matrices, 1932, p. 146. 
CoRollary. The 2-way matrices $B_{1}, \cdots, B_{n-1}$ of order $n$ are equivalent under similar transformation in $\phi$ to $\xi_{1}, \cdots, \xi_{n-1}$, if and only if $b_{i 11}^{\prime}=B_{i 12}^{\prime}=B_{i 21}^{\prime}=0$, $i=2,3, \cdots, n-1$, and the set $B_{222}^{\prime}, B_{322}^{\prime}, \cdots, B_{n-1,22}^{\prime}$ is equivalent under similar transformation in $\phi$ to the set $\xi_{2}^{\prime}, \xi_{3}^{\prime}, \cdots, \xi_{n-1}^{\prime}$ where $\xi_{i}^{\prime}, i=2, \cdots, n-1$, are $(n-1)$ by $(n-1)$ diagonal matrices which possess the $(i-1)$ st diagonal element as the only non-vanishing element, it being unity.

Let $\delta_{1}, \delta_{2}, \cdots, \delta_{m-1}$ now denote the matrices $\left(\delta_{1 i_{1} \ldots i_{p}}\right) \times\left(\delta_{j_{1}} \ldots j_{p}\right)$, $\left(\delta_{2 i_{1} \cdots i_{p}}\right) \times\left(\delta_{j_{1} \cdots j_{p}}\right), \cdots,\left(\delta_{m-1, i_{1} \cdots i_{p}}\right) \times\left(\delta_{j_{1} \cdots j_{p}}\right)$ respectively, where $\left(\delta_{j_{1} \cdots i_{p}}\right)$ is a $\delta$-matrix on $\left(j_{1}, \cdots, j_{p}\right)$ of order $t$, and $\left(\delta_{1, i_{1} \cdots i_{p}}\right), \cdots,\left(\delta_{m-1, i_{2} \cdots i_{p}}\right)$ are $i$-layers of a $\delta$-matrix on $\left(i, i_{1} \cdots i_{p}\right)$ of order $m$ obtained by setting $i=1, \cdots, m-1$ respectively. Let $B_{1}, \cdots, B_{m-1}$ be $p$-way matrices, $p \geqq 3$, of order $n$, where $n=m t$, and where the matrix $B_{1}=\left(b_{k_{1}} \ldots k_{p}\right)$ is non-singular. Let the matrices $C_{s}=\left(c_{k_{s} i_{s} j_{s}}^{s}\right) ; s=1, \cdots, p ; k_{s}=1, \cdots, n, i_{s}=1, \cdots, m$; $j_{s}=1, \cdots, t$, non-singular on $k_{s}, i_{s} j_{s}\left(i_{s} j_{s}\right.$ is a single partition), reduce $B_{1}$ to $\delta$ under similar transformation, where $\delta$ is a $\delta$-matrix on $\left(i_{1} j_{1}, i_{2} j_{2}, \cdots, i_{p} j_{p}\right)$; let these matrices simultaneously reduce $B_{2}, \cdots, B_{m-1}$ to $B_{2}^{\prime}, \cdots, B_{m-1}^{\prime}$. With these notations we can state

THEOREM 18. The set of $p$-way matrices $B_{1}, \cdots, B_{m-1}, p \geqq 3$, of order $n$ is equivalent in $\phi$ under similar transformation on $k_{1}, \cdots, k_{p}$ with $C_{1}=\left(c_{k_{1} i_{1} j_{1}}^{(1)}\right)$, $\cdots, C_{p}=\left(c_{k_{p} p_{p} j_{p}}^{(p)}\right)$ to a set $\delta_{1}, \cdots, \delta_{m-1}$ if and only if $B_{s}^{\prime}=\delta_{s}$ for $s=2, \cdots$, $m-1$ or $B_{s}^{\prime \prime}=\delta_{s}$ where $B_{2}^{\prime \prime}, \cdots, B_{m-1}^{\prime \prime}$ are obtained from $B_{2}^{\prime}, \cdots, B_{m-1}^{\prime}$ by simultaneously rearranging the $\left(i_{1} j_{1}, i_{2} j_{2}, \cdots, i_{p} j_{p}\right)$ diagonal elements of $B_{2}^{\prime}, \cdots, B_{m-1}^{\prime}$.

It has been shown in Theorem 2 that under similar transformation on the partitions $i_{1} j_{1}, i_{2} j_{2}, \cdots, i_{p} j_{p}$ the $\left(i_{1} j_{1}, i_{2} j_{2}, \cdots, i_{p} j_{p}\right)$ diagonal elements of a matrix are at most rearranged, whence Theorem 18 follows.

Let $\xi_{1}, \cdots, \xi_{n}$ now denote the $i$-layers of a $\delta$-matrix on $\left(i, k_{1}, \cdots, k_{p}\right)$ of order $n$. Let matrices $B_{s}=\left(b_{i_{1} i_{2}} \cdots i_{p}\right), s=1, \cdots, n-1$, be of order $n$ with elements in a field $\phi$. We have the

Corollary. The set of p-way matrices $B_{1}, \cdots, B_{n-1}, p \geqq 3$, of order $n$ is equivalent under similar transformation in $\phi$ on $i_{1}, \cdots, i_{p}$ with matrices $\left(c_{i_{1} k_{1}}^{(1)}\right), \cdots,\left(c_{i_{p} k_{p}}^{(p)}\right)$ to $\xi_{1}, \cdots, \xi_{n-1}$ if and only if $B_{i}=\xi_{i}$ for $i=1, \cdots, n-1$ or $B_{i}^{\prime \prime}=\xi_{i}, i=1, \cdots, n-1$, where $B_{i}^{\prime \prime}, \cdots, B_{n-1}^{\prime \prime}$ are obtained by simultaneously reordering the $\left(i_{1}, \cdots, i_{p}\right)$ diagonal elements of $B_{1}, \cdots, B_{n-1}$.

We now prove 
THEOREM 19. Given a matrix $B=\left(b_{i k_{1}} \ldots k_{p}\right)$ with elements in a field $\phi$ where $i=1, \cdots, m$ and $k_{1}, \cdots, k_{p}=1, \cdots, n=m t$. Let $B_{1}, \cdots, B_{m}$ be the $i$-layers of $B$ obtained by letting $i=1, \cdots, m$ respectively. Let $\delta$ be a $\delta$-matrix on $\left(j_{1}, \cdots, j_{p}\right)$ of order $t$, and $\delta_{1}^{\prime \prime}, \cdots, \delta_{m}^{\prime \prime}$ be the i-layers of a $\delta$-matrix $\delta^{\prime \prime}$ on $\left(i, i_{1}, \cdots, i_{p}\right)$ of order $m$ obtained by letting $i=1, \cdots, m$ respectively. The matrix $B$ is the multiple composite on $i, j$ of matrices $A_{s}=\left(a_{i j k_{s}}^{s}\right), s=1, \cdots, p$, non-singular on $i j, k_{s}$, with elements in $\phi$ if and only if there exist matrices $C_{s}=\left(c_{k_{s} i_{s} j_{s}}^{s}\right)$ non-singular on $k_{s}, i_{s} j_{s}$ with elements in $\phi$ such that

$$
\begin{aligned}
& B_{1}\left|k_{1} k_{2} \cdots k_{p}\right| C_{1} \times C_{2} \times \cdots \times C_{p}=\delta_{1}^{\prime \prime} \times \delta, \\
& \cdot . \cdots \times k_{p} \mid C_{1} \times C_{2} \times \cdots \times C_{p}=\delta_{m}^{\prime \prime} \times \delta . \\
& B_{m} \mid k_{1} k_{2} \cdots k .
\end{aligned}
$$

If $C=\left(a_{i j k_{1}}^{(1)} \cdots a_{i j k_{p}}^{(p)}\right), i$ not summed, where $A_{s}=\left(a_{i_{s} j_{s} k_{s}}^{s}\right) ; i=i_{s}=1, \cdots, m$; $j=j_{s}=1, \cdots, t ; k_{1}, \cdots, k_{p}=1, \cdots, n=m t$, then

$$
C=\delta^{\prime \prime} \times \delta\left|i_{1} \cdots i_{p} j_{1} \cdots j_{p}\right| A_{1} \times \cdots \times A_{p} .
$$

If the matrix $B=\left(b_{i k_{1}} \ldots k_{p}\right)=C$, then by (23)

$$
\begin{aligned}
B \mid k_{1} & \cdots k_{p} \mid A_{1}^{-1} \times \cdots \times A_{p}^{-1} \\
& =\delta^{\prime \prime} \times \delta\left|i_{1} \cdots i_{p} j_{1} \cdots j_{p}\right|\left(\delta_{i_{1} i_{1} i_{1}^{\prime} i_{1}}\right) \times \cdots \times\left(\delta_{i_{p} i_{p} i_{p}^{\prime} j_{p}}\right),
\end{aligned}
$$

where $A_{s}^{-1}=\left(A_{k_{s} i_{s}^{\prime} i_{s}}\right)$ is the inverse of the matrix $A_{s}$ on $i_{s} j_{s}, k_{s}$ and $\left(\delta_{i_{s} j_{s} i_{s^{\prime}} j_{s^{\prime}}}\right)$, displayed as $\left(\delta_{T_{s} T_{s}^{\prime}}\right), T_{s}=i_{s} j_{s}, T_{s}^{\prime}=i_{s}^{\prime} j_{s}^{\prime}$, is a Kronecker delta.

The author has shown* that

$$
\begin{aligned}
& \left(\delta_{i_{s} j_{s} i_{s^{\prime}} j_{s^{\prime}}}\right)=\left(\delta_{i_{s} i_{s}^{\prime}}\right) \times\left(\delta_{i_{a} j_{s^{\prime}}}\right) \\
& (s=1, \cdots, p),
\end{aligned}
$$

where $\left(\delta_{i_{s} i_{s^{\prime}}}\right),\left(\delta_{i_{s} j_{s^{\prime}}}\right)$ are Kronecker deltas. By (24) and (25) we have

$$
B\left|k_{1} \cdots k_{p}\right| A_{1}^{-1} \times \cdots \times A_{p}^{-1}=\delta^{\prime \prime} \times \delta .
$$

Equation (26) is equivalent to the set of equations

$$
\begin{aligned}
& B_{1}\left|k_{1} \cdots k_{p}\right| A_{1}^{-1} \times \cdots \times A_{p}^{-1}=\delta_{1}^{\prime \prime} \times \delta, \\
& B_{m}\left|k_{1} \cdots k_{p}\right| A_{1}^{-1} \times \cdots \times A_{p}^{-1}=\delta_{m}^{\prime \prime} \times \delta,
\end{aligned}
$$

where $\delta_{1}^{\prime \prime}, \cdots, \delta_{m}^{\prime \prime}$ are the $i$-layers of $\delta^{\prime \prime}$. This proves the theorem.

To show that the factorization property of Theorem 19 can be recognized by Theorems 17 and 18 , we note that if the $i$-layers $B_{1}, \cdots, B_{m}$ of $B$ are equivalent under non-singular linear transformations to $\delta=\delta_{1}^{\prime \prime} \times \delta, \cdots, \delta_{m}=\delta_{m}^{\prime \prime} \times \delta$

* Composition and rank of $n$-way matrices and multilinear forms, Annals of Mathematics, vol. 35 (1934), p. 629. 
as in (27), then $\bar{B}=B_{1}+\cdots+B_{m}, B_{1}, \cdots, B_{m-1}$ are equivalent under nonsingular linear transformations to $\delta=\left(\delta_{1}^{\prime \prime} \times \delta+\cdots+\delta_{m}^{\prime \prime} \times \delta\right), \delta_{1}, \cdots, \delta_{m-1}$, where $\delta_{1}, \cdots, \delta_{m-1}$ are defined as in Theorem 18, and conversely. Now $\delta$ is a $\delta$-matrix on the partitions $\left(i_{1} j_{1}, \cdots, i_{p} j_{p}\right)$. If $\bar{B}$ is non-singular, reduce $\bar{B}$ to $\delta$ under non-singular linear transformations. Simultaneously $B_{1}, \cdots, B_{m-1}$ transform into matrices $B_{1}^{\prime}, \cdots, B_{m-1}{ }^{\prime}$. The matrices $\bar{B}, B_{1}, \cdots, B_{m-1}$ are equivalent to $\delta, \delta_{1}, \cdots, \delta_{m-1}$ if and only if $B_{1}^{\prime}, \cdots, B_{m-1}^{\prime}$ are equivalent under similar transformation to $\delta_{1}, \cdots, \delta_{m-1}$. The conditions for such equivalence for the 2-way case are given in Theorem 17 and for the $p$-way case, $p \geqq 3$, in Theorem 18.

CoRollary. Let $\xi_{1}, \cdots, \xi_{n}$ be the $i$-layers of a $\delta$-matrix on $\left(i, i_{1}, \cdots, i_{p}\right)$ of order $n$, where these layers are obtained by letting $i=1, \cdots, n$ respectively. The matrix $B=\left(b_{i k_{1}} \ldots k_{p}\right)$ of order $n$ with elements in a field $\phi$ can be written in the form $\left(a_{i k_{1}}^{(1)} \cdots a_{i k_{p}}^{(p)}\right), i$ not summed, where $\left(a_{i k_{1}}^{(1)}\right), \cdots,\left(a_{i k_{p}}^{(p)}\right)$ are non-singular and possess elements in $\phi$, if and only if the i-layers $B_{1}=\left(b_{1 k_{1}} \ldots k_{p}\right)$, $B_{2}=\left(b_{2 k_{1}} \ldots k_{p}\right), \cdots, B_{n}=\left(b_{n k_{1}} \ldots k_{p}\right)$ of $B$ are equivalent in $\phi$ to $\xi_{1}, \xi_{2}, \cdots, \xi_{n}$.

Consider the matrix $B=\left(b_{i k_{1}} \ldots k_{p}\right), p \geqq 3$, of order $m$ on $i$, and order $n=m t$ on $k_{1}, \cdots, k_{p}$. Let $\delta_{1}, \cdots, \delta_{m-1}$ be again defined as in Theorem 18. If $\bar{B}=\left(\sum_{i=1}^{m} b_{i k_{1}} \ldots k_{p}\right)$ is non-singular, the matrices $E_{s}=\left(e_{i_{s} j_{s} k_{s}}^{s}\right)$, non-singular on $k_{s}, i_{s} j_{s}$, which reduce $\bar{B}$ to a $\delta$-matrix on $\left(i_{1} j_{1}, \cdots, i_{p} j_{p}\right) ; i_{1}, \cdots, i_{p}=1, \cdots, m$; $j_{1}, \cdots, j_{p}=1, \cdots, t$, reduce the $i$-layers $B_{1}, \cdots, B_{m-1}$ of $B$, obtained by setting $i=1, \cdots, m-1$ respectively, to a set $B_{1}^{\prime}, \cdots, B_{m-1}^{\prime}$. Theorems 2, 18, and 19 imply

ThEOREM 20. For $B=\left(b_{i k_{1}} \cdots k_{p}\right), p \geqq 3$, of order $m$ on $i$ and $n=m t$ on $k_{1}, \cdots, k_{p}$, to be the multiple composite on $i, j$ of the matrices $A_{s}=\left(a_{i j k_{s}}^{s}\right)$, $s=1, \cdots, p$, non-singular on $i j, k_{s}$, it is necessary that the sum $\bar{B}$ of the $i$-layers of $B$ be non-singular. It is further necessary that

$$
B_{1}^{\prime}=\delta_{1}, \cdots, B_{m-1}^{\prime}=\delta_{m-1},
$$

or

$$
B_{1}^{\prime \prime}=\delta_{1}, \cdots, B_{m-1}^{\prime \prime}=\delta_{m-1},
$$

where $B_{1}^{\prime \prime}, \cdots, B_{m-1}^{\prime \prime}$ are obtained from $B_{1}^{\prime}, \cdots, B_{m-1}^{\prime}$ by simultaneous rearrangements of the $\left(i_{1} j_{1}, \cdots, i_{p} j_{p}\right)$ diagonal elements of $B_{1}^{\prime}, \cdots, B_{m_{-1}^{\prime}}^{\prime}$. Theise conditions are also sufficient.

Now let $B$ represent the matrix $\left(b_{i k_{1} \cdots k_{p}}\right), p \geqq 3$, of order $n$. Let the $i$ layers of $B$ obtained by setting $i=1, \cdots, n$ be denoted by $B_{1}, \cdots, B_{n}$ respectively. If $\bar{B}=\left(\sum_{i=1}^{n} b_{i k_{1} \cdots k_{p}}\right)$ is non-singular, reduce $\bar{B}, B_{1}, \cdots, B_{n-1}$ by 
means of non-singular linear transformations to $\xi, B_{1}^{\prime}, \cdots, B_{n-1}^{\prime}$, where $\xi$ is a $\delta$-matrix on $\left(i_{1}, \cdots, i_{p}\right)$ of order $n$. As in the corollary of Theorem 18 let $\xi_{1}, \cdots, \xi_{n}$ be the $i$-layers of a $\delta$-matrix on $\left(i, i_{1}, \cdots, i_{p}\right)$ of order $n$. We have the following

Corollary. For $B=\left(b_{i k_{1} \ldots k_{p}}\right), p \geqq 3$, of order $n$ with elements in $a$ field $\phi$ to be factorable into the form $K=\left(a_{i k_{1}}^{(1)} \cdots a_{i k_{p}}^{(p)}\right)$, $i$ not summed, where $\left(a_{i k_{1}}^{(1)}\right), \cdots\left(a_{i k_{p}}^{(p)}\right)$ are non-singular with elements in $\phi$, it is necessary that $\bar{B}$ be non-singular. It is further necessary that $B_{s}^{\prime}=\xi_{s} ; s=1, \cdots, n-1$, or $B_{s}^{\prime \prime}=\xi_{s} ; s=1, \cdots, n-1$, where $B_{1}^{\prime \prime}, \cdots, B_{n-1}^{\prime \prime}$ are obtained from $B_{1}^{\prime}, \cdots, B_{n-1}^{\prime}$ by simultaneous rearrangements of the $\left(i_{1}, \cdots, i_{p}\right)$ diagonal elements of $B_{1}^{\prime}, \cdots, B_{n-1}^{\prime}$. These conditions are also sufficient.

Theorems 17 to 20 may be extended at once to the multiple composite on $i, j$ of $A_{1}=\left(a_{i j k_{1}}^{(1)}\right), \cdots, A_{p}=\left(a_{i j k_{p}}^{(p)}\right)$ where $A_{1}, \cdots, A_{p}$ are non-singular on $i j$ only.

The matrix $\delta^{\prime \prime} \times \delta$ above is a canonical matrix of a class of $(p+1)$-way matrices. We have hence determined necessary and sufficient conditions for the equivalence of a $(p+1)$-way matrix to such a diagonal matrix under nonsingular linear transformations on all but one index.

\section{APPENDIX}

For certain situations, the equivalence under similar transformation in a given field of a set of 2-way matrices $S=\left(T_{1}, \cdots, T_{m}\right)$ of order $n$ to a set of diagonal matrices can be recognized by the rank of a matrix $H$ associated with $S$. This appendix is devoted to the derivation of $H$.

Let

$$
C_{i}=\left(\begin{array}{lll}
c_{1}{ }^{i} & & \\
& \ddots & 0 \\
0 & \ddots & \\
c_{n}{ }^{i}
\end{array}\right)
$$

be a classical canonical matrix $\dagger$ to which $T_{i}$ is similar for $i=1, \cdots, m$.

Let $\alpha_{i}, i=1, \cdots, n$, denote the matrix

$$
\left(\begin{array}{c}
T_{1}^{\prime}-d_{i}^{1} I \\
\vdots \\
T_{m}^{\prime}-d_{i}^{m} I
\end{array}\right)
$$

where $T_{1}^{\prime}, \cdots, T_{m}^{\prime}$ are the transposes of $T_{1}, \cdots, T_{m}$ and $d_{1}^{\sigma}, \cdots, d_{n}^{\sigma}$ are permutations of $c_{1}{ }^{\circ}, \cdots, c_{n^{*}}$ for $\sigma=1, \cdots, m$. $I$ is a Kronecker delta. Assume that the matrices $\alpha_{i}, i=1, \cdots, n$, are all of rank $n-1$. Then there exist

$\dagger i$ in $c_{j}{ }^{i}$ is a superscript. 
values $\psi_{i 1}, \psi_{i 2}, \cdots, \psi_{i, n(m-1)+1}$ of $\psi_{i}$ and a value of $\xi_{i}$ such that the minor $A_{i 1}, i=1, \cdots, n$, obtained from $\alpha_{i}$ by deleting the $\psi_{i 1}$ th, $\psi_{i 2}$ th, $\cdots, \psi_{i, n(m-1)+1}$ th rows and the $\xi_{i}$ th column is a non-singular minor of order $n-1$. Let $A_{2 i}$, $i=1, \cdots, n$, be the $\xi_{i}$ th column of $\alpha_{i}$ with the $\psi_{i 1}$ th, $\psi_{i 2}$ th, $\cdots, \psi_{i, n(m-1)+1}$ th elements deleted. Let $H_{11}, \cdots, H_{1 n}, H_{21}, \cdots, H_{2 n}$ be defined by the relations

$$
\left(\begin{array}{l}
H_{11} \\
H_{21}
\end{array}\right)=-A_{11}^{-1} A_{21}, \cdots,\left(\begin{array}{c}
H_{1 n} \\
H_{2 n}
\end{array}\right)=-A_{1 n}^{-1} A_{2 n},
$$

where $H_{1 i}$ is a column composed of the first $\xi_{i}-1$ elements of the column $-A_{1 i}{ }^{-1} A_{2 i}$, and $H_{2 i}$ is a column composed of the remaining elements of $-A_{1 i}^{-1} A_{2 i}$. Let $H$ now denote the matrix

$$
\left(\begin{array}{lll}
H_{11} & & H_{1 n} \\
1 & \cdots & 1 \\
H_{21} & & H_{2 n}
\end{array}\right),
$$

where the unit element in the $i$ th column of $H$ occurs in the $\xi_{i}$ th row for $i=1, \cdots, n$.

Evidently the set $S$ is equivalent under similar transformation to a set of diagonal matrices if and only if the set $S$ is equivalent under similar transformation to the set $S^{\prime}=\left(C_{1}, \cdots, C_{m}\right)$ or $S^{*}=\left(C_{1}^{*}, \cdots, C_{m}^{*}\right)$, where $C_{1}^{*}, \cdots, C_{m}^{*}$ are matrices obtained from $C_{1}, \cdots, C_{m}$ by arbitrary interchanges of the diagonal elements. If the set $S$ is to be equivalent under similar transformation to the set $S^{\prime}$, there exists a non-singular matrix $X=\left(x_{i j}\right)$ such that

$$
X T_{1} X^{-1}=C_{1}, \cdots, X T_{m} X^{-1}=C_{m},
$$

or what is the same thing,

$$
X T_{1}=C_{1} X, \cdots, X T_{m}=C_{m} X .
$$

Equations (1) are equivalent to the set of equations

$$
\begin{gathered}
\alpha_{1}\left(\begin{array}{c}
x_{11} \\
\vdots \\
x_{1}
\end{array}\right)=0, \\
\cdot \cdot \cdot \\
\alpha_{n}\left(\begin{array}{c}
x_{n 1} \\
\vdots \\
x_{n}
\end{array}\right)=0 .
\end{gathered}
$$


Since $\alpha_{1}$ is of rank $n-1$, equation $\left(2_{1}\right)$ can be written as

$$
A_{11}\left(\begin{array}{l}
x_{11} \\
\vdots \\
x_{1, \xi_{1}-1} \\
x_{1, \xi_{1}+1} \\
\vdots \\
x_{1 n}
\end{array}\right)=-A_{21} x_{1, \xi_{1}} \text {. }
$$

Now

$$
\left(\begin{array}{l}
x_{11} \\
\vdots \\
x_{1, \xi_{1}-1} \\
x_{1, \xi_{1}+1} \\
\vdots \\
x_{1 n}
\end{array}\right)=-A_{11}^{-1} A_{21} x_{1, \xi_{1}}
$$

Since $\alpha_{2}, \cdots, \alpha_{n}$ are all of rank $n-1$, we obtain similar solutions from $\left(2_{2}\right), \cdots,\left(2_{n}\right)$ of the form

$$
\left(\begin{array}{l}
x_{21} \\
\vdots \\
x_{2, \xi_{2}-1} \\
x_{2, \xi_{2}+1} \\
\vdots \\
x_{2 n}
\end{array}\right)=-A_{12}^{-1} A_{22} x_{2, \xi_{2}}, \cdots,\left(\begin{array}{l}
x_{n 1} \\
\vdots \\
x_{n, \xi_{n}-1} \\
x_{n, \xi_{n}+1} \\
\vdots \\
x_{n n}
\end{array}\right)=-A_{1 n}^{-1} A_{2 n} x_{n, \xi_{n}} \text {. }
$$

The matrix $X$ can now be written as

$$
X=\left(\begin{array}{rrr}
H_{11} x_{1, \xi_{1}} & & H_{1 n} x_{n, \xi_{n}} \\
x_{1, \xi_{1}} & \cdots & x_{n, \xi_{n}} \\
H_{21} x_{1, \xi_{1}} & & H_{2 n} x_{n, \xi_{n}}
\end{array}\right) .
$$

Evidently

$$
X=H\left(\begin{array}{ccc}
x_{1, \xi_{1}} & & \\
& & 0 \\
0 & \ddots & \\
& & x_{n, \xi_{n}}
\end{array}\right),
$$

whence $X$ can be taken non-singular if and only if $H$ is non-singular. 
A like argument holds if the set $S^{\prime}$ is replaced in the equations of (1) by $S^{*}$. We have proved the

THEOREM. The set $S$ is equivalent under similar transformation to a set of diagonal matrices if and only if $H$ is non-singular for at least one choice of the quantities $d_{1}^{1}, \cdots, d_{n}^{1}, d_{1}{ }^{2}, \cdots, d_{n}{ }^{2}, \cdots, d_{1}{ }^{m}, \cdots, d_{n}{ }^{m}$.

Armour Institute of Technology, Chicago, Ill. 